\title{
Fluoxetina:
}

\section{profilo terapeutico e farmacoeconomico di un prodotto generico}

Carlo Della Pepa*, Mario Eandi*

\section{ABSTRACT}

Fluoxetine has been the first antidepressive agent of the class of selective serotonine reuptake inhibitors (SSRI) to be introduced in therapy, back in 1987. The availability of SSRIs has meant a great advance in the treatment of major depression and other neuro-psychiatric pathologies.

In this paper we review the scientific and clinical research on fluoxetine that has been published in the last six years, as the prior work has been extensively examined in a review conducted on the tenth anniversary of its introduction on the market. The review focuses on cost minimization strategies that can be conducted following the introduction of low cost generic fluoxetine.

Farmeconomia e percorsi terapeutici 2003; 4 (3): 125-152

\section{PREMESSE}

La fluoxetina è il capostipite dei farmaci inibitori selettivi della ricaptazione di serotonina (SSRI). Questa molecola ha rappresentato una svolta significativa nella terapia farmacologia della depressione maggiore, grazie al migliorato rapporto rischio/beneficio nei confronti dei triciclici, ed ha trovato utile impiego anche in diverse altre patologie neuropsichiatriche e principalmente nei disturbi ossessivo-compulsivi, nella bulimia nervosa, nel disturbo disforico premestruale. Un esaustivo profilo farmacologico, terapeutico e farmacoeconomico della fluoxetina è stato pubblicato nel 1997, in occasione del decimo anniversario della sua introduzione nell' armamentario terapeutico [1]. Da allora lo sviluppo scientifico della fluoxetina è continuato ininterrottamente fino ai giorni nostri, fornendo ulteriori elementi di valutazione della sua efficacia e tollerabilità ed ampliando il numero delle indicazioni possibili.

In uso clinico da oltre 16 anni come prodotto di marchio (branded), la fluoxetina è da pochi anni disponibile in Italia anche come prodotto generico.

In particolare le confezioni di prodotti generici orali di fluoxetina ( 12 e 28 capsule da 20 $\mathrm{mg}, 12$ e 28 compresse da $20 \mathrm{mg}$, soluzione 60 $\mathrm{ml} 20 \mathrm{mg} / 5 \mathrm{ml}$ ) hanno un prezzo di vendita nettamente inferiore a quello degli equivalenti prodotti branded e sono assunti come "prezzo di riferimento" rimborsabile dal SSN. Inoltre il prezzo dei prodotti generici di fluoxetina è net- tamente inferiore anche a quello degli altri SSRI e di altri antidepressivi recenti, pur risultando terapeuticamente equivalenti nella maggior parte delle indicazioni cliniche.

I prodotti "generici" sono copie di un prodotto farmaceutico maturo (detto anche "innovatore") ma ormai fuori brevetto e come questo sono specialità farmaceutiche a tutti gli effetti, che ottengono l'autorizzazione all'immissione in commercio (AIC), ossia la registrazione, dal Ministero della Salute secondo procedure abbreviate [2].

Il farmaco "generico" ha la stessa composizione in principi attivi, una forma farmaceutica equivalente, le stesse indicazioni, lo stesso dosaggio e la stessa via di somministrazione del prodotto innovatore.

Per registrare un "generico" è sufficiente presentare al Ministero della Salute una domanda di AIC, accompagnata dalla documentazione descrittiva dello sviluppo formulativo, del processo produttivo, della dimostrazione di equivalenza farmaceutica e di bioequivalenza con il prodotto "innovatore".

I prodotti farmaceutici "copia" possono essere registrati e commercializzati con un nome di fantasia ("generici branded", non distinguibili da una qualsiasi altra specialità medicinale) o con la denominazione comune internazionale (DCI) seguita dal nome del produttore ("generici unbranded" o semplicemente "generici"). Alcune specialità branded di fluoxetina orale registrate in Italia sono in realtà copie dell' originatore e possono essere considerati "generici

\section{* Dipartimento di Farmacologia, Università di Torino}


branded": attualmente questi prodotti hanno un prezzo inferiore a quello dell'originatore, ma superiore a quello del generico.

I cardini della politica dei "generici" in Italia sono essenzialmente tre: 1) il prezzo di un "generico" non può essere superiore all' $80 \%$ del prezzo del prodotto "innovatore", mentre un produttore può fissarlo ad un livello anche notevolmente inferiore, innescando in tal modo una dinamica di prezzi al ribasso grazie alla possibilità di entrare in un mercato concorrenziale; 2) il prezzo del generico, o il prezzo più basso tra quelli dei prodotti generici equivalenti, viene assunto come prezzo di riferimento rimborsabile dal SSN per quel farmaco; 3) il farmacista può, anzi deve, dispensare un "generico" in sostituzione di un equivalente prodotto di marchio, salvo il caso in cui il medico specifichi il contrario o il paziente sia disposto a pagare l'eventuale differenza rispetto alla quota rimborsata dal SSN.

La disponibilità di prodotti generici orali di fluoxetina, equivalenti agli analoghi prodotti di marchio ma venduti ad un prezzo nettamente inferiore, consente di realizzare una strategia di minimizzazione dei costi, con risparmi sensibili per il SSN e per il paziente, senza riduzione di efficacia clinica.

Analogamente, la dimostrazione comparativa diretta dell'equivalenza terapeutica tra fluoxetina ed altri farmaci antidepressivi costituisce la base documentale per poter applicare la logica della minimizzazione dei costi e indicare i prodotti generici di fluoxetina come quelli oggi più convenienti per il SSN e per i pazienti.

In questo lavoro presentiamo un aggiornamento del profilo farmacologico clinico e terapeutico della fluoxetina, analizzando i principali risultati delle ricerche cliniche pubblicate negli ultimi 6 anni. I risultati più significativi verranno discussi considerando soprattutto sia le conseguenze sanitarie correlabili ad un rinnovato interesse per la fluoxetina sia le ricadute economiche per il SSN e per i pazienti derivanti dalla disponibilità di prodotti generici di fluoxetina sul mercato italiano.

\section{FARMACOLOGIACLINICA}

\section{Selettività e meccanismo d'azione}

I farmaci antidepressivi attualmente in uso inducono un incremento della disponibilità sinaptica di noradrenalina (NA), dopamina (DA) e/o serotonina (5HT), sia bloccando la ricaptazione neuronale di questi trasmettitori, sia riducendone il catabolismo mediante l'inibizione delle MAO, sia rimuovendo il tono inibitorio sul rilascio o sull' attività neuronale.

La fluoxetina è un farmaco inibitore selettivo della ricaptazione di serotonina (SSRI). La Tabella 1 evidenzia la selettività d'azione della fluoxetina e degli altri SSRI sulla ricaptazione della serotonina, fattore che li differenzia dai classici triciclici, considerati inibitori non selettivi della ricaptazione di noradrenalina e di serotonina.

Il meccanismo d'azione della fluoxetina a livello della sinapsi serotoninergica è multiplo e si differenzia in una fase acuta e in una fase cronica. L'azione acuta della fluoxetina consiste nell'aumento delle concentrazioni sinaptiche di serotonina dovuto sia al blocco selettivo della ricaptazione sia alla facilitazione del rilascio di serotonina provocato dall' antagonismo degli autorecettori 5HT1 pre-sinaptici.

L'azione cronica della fluoxetina consiste principalmente nella down-regulation dei recettori serotoninergici post-sinaptici e dal correlato adattamento della cascata dei secondi messaggeri, che in ultima analisi possono determinare una modificazione dell' espressione genica dei fattori di crescita neuronali.
Tabella 1

Inibizione dei trasportatori umani di noradrenalina (NA-T), serotonina $(5-H T-T) e$ dopamina $(D A-T)$ da parte della fluoxetina e di alcuni altri antidepressivi a differente selettività sulla ricaptazione della serotonina o della noradrenalina. (Dati adattati da: Frazer, 1997; Owens et al, 1997; Leonard \& Richelson, 2000) [3-5]

\begin{tabular}{|c|c|c|c|c|}
\hline Farmaco & \multicolumn{3}{|c|}{ Costante di inibizione (Ki) dei trasportatori di monoamine* } & $\begin{array}{c}\text { Selettività 5-HT } \\
\text { (NE-T/5-HT-T) }\end{array}$ \\
\hline Fluoxetina & 244 & 0,81 & 3.600 & 301 \\
\hline Norfluoxetina & 410 & 25 & 1.100 & 16,40 \\
\hline Fluvoxamina & 1.300 & 2,22 & 9.100 & 586 \\
\hline Paroxetina & 40 & 0,125 & 500 & 320 \\
\hline Sertralina & 417 & 0,293 & 25 & 1423 \\
\hline Citalopram & 400 & 1,16 & 28.000 & 345 \\
\hline Venlafaxina & 1.060 & 9,1 & 9.100 & 117 \\
\hline Reboxetina & 7,14 & 58,8 & 11.500 & 0,12 \\
\hline Desipramina & 0,83 & 17,5 & 3.200 & 0,05 \\
\hline Imipramina & 37 & 1,41 & 8.300 & 26,24 \\
\hline Amitriptilina & 34,5 & 4,33 & 3.200 & 7,97 \\
\hline Clomipramina & 37 & 0,28 & 2.200 & 132 \\
\hline Bupropion & 52.600 & 9.100 & 526 & 5,78 \\
\hline
\end{tabular}

* Potenza espressa come costante di inibizione (Ki) in nM, stimata in base a test di competizione su membrane di linee cellulari transfettate con i geni umani delle specifiche proteine di trasporto 
I meccanismi di regolazione e modulazione a lungo termine delle funzioni sinaptiche correlano in modo convincente con l'evoluzione temporale della risposta terapeutica osservata nel paziente depresso. La pratica clinica, infatti, indica chiaramente che la risposta terapeutica antidepressiva alla fluoxetina e agli altri farmaci antidepressivi si manifesta dopo almeno 2-3 settimane di trattamento, segno evidente che si tratta di un effetto cronico e acuto del farmaco.

Il sistema serotoninergico partecipa al controllo di molteplici funzioni regolate dal sistema nervoso centrale (SNC) e la sua disregolazione è stata chiamata in causa in diverse patologie psichiatriche e comportamentali, in particolare, oltre che nei disturbi dell'umore, anche nei disturbi d'ansia (disturbo ossessivo-compulsivo, disturbo d'ansia generalizzata, disturbo di panico), nei disturbi del comportamento alimentare e del comportamento sessuale e nel dolore cronico. La disponibilità di farmaci inibitori selettivi della ricaptazione di serotonina, come la fluoxetina, ha contribuito in modo determinante a chiarire le basi neurochimiche di questi disturbi e, d'altra parte, l'ipotesi serotoninergica ha spinto i ricercatori a documentare l'efficacia della fluoxetina non solo nella depressione maggiore, ma anche in molti altri disturbi, diversi e indipendenti dalla depressione.

La maggior differenza clinica tra i vari antidepressivi riguarda il profilo degli effetti collaterali e delle reazioni avverse, profilo correlabile alla specificità dei meccanismi neurochimici di ogni singola molecola, più che alla selettività d'azione.

Infatti, ai fini del profilo di tollerabilità risulta essere più importante la specificità delle singole molecole, ossia l'assenza d'interazioni con sistemi recettoriali o neurochimici non implicati nell'azione antidepressiva ma responsabili di eventuali effetti collaterali o reazioni avverse.
La fluoxetina e gli altri farmaci SSRI presentano un'affinità di legame estremamente bassa o nulla per i recettori a-adrenergici, muscarinici e istaminergici, come dimostra la Tabella 2.

\section{Farmacocinetica}

Le principali caratteristiche farmacocinetiche differenziali della fluoxetina consistono nella sua lunga emivita (2-3 giorni), nella formazione di un metabolita attivo, la norfluoxetina, ad emivita protratta di 14-21 giorni, e nel coinvolgimento prevalente dei citocromi CYP2C19 e CYP2D6 nel suo metabolismo. La lunga emivita della fluoxetina e l'emivita ancora più lunga del suo metabolita attivo giustificano la sua prolungata durata d'azione e la minor frequenza di sintomi d'astinenza dopo la sospensione del trattamento, rispetto agli altri SSRI.

La fluoxetina è ben assorbita a livello intestinale e la sua biodisponibilità orale è pressoché totale [6]. Dopo singola dose orale il picco di concentrazione plasmatici della fluoxetina avviene entro 4-8 ore dalla somministrazione mentre il picco della norfluoxetina compare dopo circa 76 ore [6-8].

La fluoxetina è legata per oltre il $94 \%$ all'albumina e all'a1-glicoproteina: tale legame farmaco-proteico non risulta alterato nei pazienti con insufficienza renale $[6,8]$.

Il volume apparente di distribuzione $(\mathrm{Vd})$ è molto elevato e varia tra 1000 e $7200 \mathrm{~L}$ mentre il corrispettivo Vd della norfluoxetina è compreso tra 700 e $5700 \mathrm{~L}$. Il volume di distribuzione della fluoxetina o della norfluoxetina non è correlato con la funzionalità renale [8]. Dato un volume di distribuzione così elevato, le concentrazioni plasmatiche del farmaco e del suo metabolita sono molto basse. Dopo somministrazione di $60 \mathrm{mg}$ di fluoxetina per 5 settimane, sono state misurate concentrazioni plasmatiche medie di fluoxetina e di

\begin{tabular}{|c|c|c|c|c|c|c|c|c|c|}
\hline Farmaco & $\alpha_{1}$ & $\alpha_{2}$ & $\beta$ & $\mathbf{D}_{2}$ & $5 \mathrm{HT}_{1}$ & $5 \mathrm{HT}_{2}$ & $\mathbf{H}_{1}$ & Ach & Effetti collaterali \\
\hline Fluoxetina & - & - & - & - & - & - & - & + & Disturbi GI (nausea, vomito, \\
\hline Fluovoxamina & - & - & - & - & - & - & - & - & diarrea, anoressia). Ansia, \\
\hline Sertralina & ++ & - & - & - & - & - & - & + & tremori, insonnia, debolezza. \\
\hline Paroxetina & - & - & - & \pm & - & - & - & ++ & Disturbi sessuali \\
\hline Citalopram & - & - & - & - & - & - & - & + & \\
\hline Venlafaxina & - & - & - & - & - & - & - & - & $\begin{array}{l}\text { Ansia, sedazione, } \\
\text { ipertensione, nausea }\end{array}$ \\
\hline Reboxetina & - & - & - & \pm & \pm & - & - & - & $\begin{array}{l}\text { Secchezza fauci, stipsi, } \\
\text { sudorazione, insonnia }\end{array}$ \\
\hline Desipramina & ++ & ++ & - & + & + & +++ & +++ & +++ & Tachicardia, aritmie, \\
\hline Imipramina & ++ & + & - & ++ & + & ++ & ++ & +++ & ipotensione, tremori, \\
\hline Amitriptilina & + & - & - & + & + & ++ & + & +++ & sedazione, confusione, \\
\hline Clomipramina & ++ & + & - & + & \pm & ++ & ++ & +++ & $\begin{array}{l}\text { aumento peso, secchezza } \\
\text { fauci, stipsi, ritenzione urinaria, }\end{array}$ \\
\hline
\end{tabular}

Tabella 2

Affinità recettoriali ed effetti collaterali di alcuni antidepressivi 
norfluoxetina allo steady-state rispettivamente di $477 \mathrm{ng} / \mathrm{mL}$ e di $393 \mathrm{ng} / \mathrm{mL}$. Queste concentrazioni ematiche sono state correlate con l'azione terapeutica antidepressiva [9].

Il metabolismo è prevalentemente epatico e avviene con il coinvolgimento di alcuni citocromi P450, in particolare il CYP2D6 ed il CYP2C19. Mediante una reazione di Ndemetilazione si forma la norfluoxetina, il metabolita attivo che presenta un'attività farmacologica analoga al progenitore, ma un'emivita prolungata. È inoltre possibile la formazione in piccole quantità di glucuronoconiugati $[6,8]$.

Un recente lavoro ha documentato l'influenza del polimorfismo genetico del citocromo CYP2C19 sul metabolismo della fluoxetina [10]. La presenza della mutazione CYP2C19*2 o CYP2C19*3 comporta un rallentamento del metabolismo nei soggetti portatori (metabolizzatori lenti) rispetto ai normali (metabolizzatori rapidi). Dopo somministrazione di $40 \mathrm{mg}$ di fluoxetina, la clearance sistemica della fluoxetina nei metabolizzatori lenti risulta inferiore del $55 \%$ rispetto a quella dei metabolizzatori rapidi. I metabolizzatori rapidi hanno le più basse concentrazioni di picco di fluoxetina e le più alte concentrazioni di norfluoxetina rispetto ai metabolizzatori lenti.

La farmacocinetica della fluoxetina e il suo effetto sul citocromo CYP2C19 sono stati studiati comparativamente su un gruppo di volontari adulti giovani (18-40 anni) e su un gruppo di anziani ( $<65$ anni), di sesso maschile [11]. I soggetti sono stati trattati per 6 settimane con fluoxetina alla dose di $20 \mathrm{mg} / \mathrm{di}$ e per ulteriori 6 settimane alla dose di 40mg/die. Allo steady-state le concentrazioni di picco (Cmax) e di valle (Cmin) e l'area sotto la curva tra le dosi (AUC0-24) della fluoxetina non sono risultate significativamente differenti negli anziani rispetto ai giovani adulti, mentre le Cmax, Cmin e AUC0-24 della norfluoxetina sono risultate inferiori di oltre il 20\% (p<0,05) negli anziani. L'emivita di eliminazione della fluoxetina e della norfluoxetina è risultata tendenzialmente più lunga di circa il $25 \%$ negli anziani rispetto ai giovani adulti, sebbene l'elevata variabilità interindividuale e la dimensio- ne del campione abbiano impedito di raggiungere una significatività statistica.

La somministrazione di $20 \mathrm{mg} / \mathrm{die}$ di fluoxetina ha indotto una significativa inibizione del CYP2C19 trasformando il 68\% dei metabolizzatori rapidi in metabolizzatori lenti o intermedi. Tale effetto è risultato ulteriormente potenziato alla dose di $40 \mathrm{mg} / \mathrm{die}$, con una conversione a metabolizzatori lenti di ben 1' $82 \%$ dei metabolizzatori rapidi [11].

La fluoxetina viene escreta per il $60 \%$ mediante la via renale, prevalentemente sotto forma di metaboliti, e per il $12 \%$ per via fecale. Nelle urine si ritrova dal $2,5 \%$ al 5,0\% della dose in forma immodificata e circa il $10 \%$ come norfluoxetina libera. I metaboliti glucuronoconiugati di fluoxetina e norfluoxetina rappresentano rispettivamente il 5,2\% e il 9,5\% della dose eliminata per via renale [6].

La fluoxetina viene escreta anche nel latte materno e può indurre una significativa esposizione dei neonati allattati al seno da madri trattate con il farmaco. In un recente studio condotto su 14 coppie madre-neonato, l'esposizione media dei bambini allattati è risultata pari al 6,81\% (3,36\% di fluoxetina e 3,45\% di norfluoxetina) [12]. Altri studi precedenti avevano documentato che nel latte materno può essere escreta una percentuale della dose di fluoxetina variabile dal 3,3\% al 10\% [13, 14].

L'emivita di eliminazione della fluoxetina è di 2-3 giorni dopo brevi somministrazioni mentre è compresa in un intervallo di 4-6 giorni dopo lunghi periodi di trattamento; l'emivita del metabolita attivo norfluoxetina è di 16-20 giorni $[6,8]$. L'emivita media nei soggetti metabolizzatori rapidi è di 28 ore mentre nei metabolizzatori lenti è di 62 ore, una media di 3,6 giorni è stata stimata nei soggetti normali (range 1-13 giorni) [10].

La Tabella 3 riporta le caratteristiche farmacocinetiche essenziali e il range posologico indicato nella scheda tecnica dei principali antidepressivi SSRI disponibili in Italia.

\section{Posologia nei pazienti con insufficienza renale}

Nessun aggiustamento posologico della fluoxetina sembra essere necessario nei pazienti
Tabella 3

Caratteristiche

farmacocinetiche, posologie giornaliere indicate nella scheda tecnica deifarmaci antidepressivi disponibili in Italia

\begin{tabular}{lccccc}
\hline Farmaco & Emivita & $\begin{array}{c}\text { Escrezione } \\
\text { renale }\end{array}$ & $\begin{array}{c}\text { Metaboliti } \\
\text { attivi }\end{array}$ & $\begin{array}{c}\text { Cinetica } \\
\text { lineare }\end{array}$ & $\begin{array}{c}\text { Range dosi } \\
\text { (mg/die) }\end{array}$ \\
\hline Fluoxetina & $\begin{array}{c}4-6 \text { gg. (Steady-State) } \\
\text { 16-20 gg. (norfluoxetina) }\end{array}$ & $60 \%$ & SI & NO & $20-80$ \\
Fluvoxamina & 15,6 ore & $94 \%$ & NO & NO & $50-300$ \\
Paroxetina & $17-22$ ore & $65-67 \%$ & NO & NO & $10-60$ \\
Sertralina & 24 ore & $40-45 \%$ & NO & SI & $25-200$ \\
Citalopram & $33-37$ ore & $20 \%$ & NO & SI & $20-60$ \\
\hline
\end{tabular}


con insufficienza renale, compresi quelli sottoposti a dialisi, dal momento che solo una quota percentuale minima della dose di fluoxetina viene eliminata nelle urine immodificata o come metabolita attivo. Dopo somministrazione di dosi singole non è stata dimostrata alcuna correlazione tra grado di insufficienza renale e velocità di eliminazione renale, volume di distribuzione o legame proteico della fluoxetina $[6,8]$. Non risulta, tuttavia, adeguatamente studiata la relazione tra insufficienza renale e trattamento a dosi ripetute e non si può escludere un incremento anomalo dell'accumulo di fluoxetina e di norfluoxetina nei casi di grave insufficienza renale [8].

La fluoxetina e la norfluoxetina, dato il loro elevato volume di distribuzione e l'elevata quota di legame proteico, hanno scarsa tendenza alla clearance extracorporea mediante dialisi. Durante sedute di dialisi le concentrazioni plasmatiche di fluoxetina e del suo metabolita attivo non subiscono variazioni significative [8]. Pertanto non è necessario aggiustare la posologia durante la dialisi.

\section{Posologia nei pazienti con insufficienza epatica}

L'aggiustamento della posologia può essere necessario nei pazienti con insufficienza epatica, poiché la fluoxetina viene estensivamente metabolizzata nel fegato [6].

Nei pazienti con cirrosi alcolica stabilizzata è stata osservata una significativa riduzione della clearance plasmatica e un corrispondente aumento dell'emivita della fluoxetina. In questi pazienti la formazione del metabolita attivo, norfluoxetina, e la sua clearance sono risultate diminuite [15].

L'aggiustamento della posologia nei pazienti affetti da insufficienza epatica può essere attuata riducendo la dose o allungando l'intervallo tra le dosi. Nei pazienti con cirrosi compensata (assenza di ascite) è raccomandata una riduzione approssimativa del $50 \%$ della posologia, mentre in pazienti cirrotici scompensati possono essere necessarie riduzioni maggiori.

\section{Posologia nei soggetti anziani e nei bambini-adolescenti}

Nei soggetti anziani è stata osservata una tendenza alla riduzione della clearance e all'allungamento dell'emivita della fluoxetina e della norfluoxetina. Pertanto nei soggetti anziani può essere necessario ridurre la posologia per evitare eccessivi accumuli [11].

Non è stata stabilita una posologia di riferimento per pazienti pediatrici con età inferiore ai 7 anni.
In soggetti di età compresa tra 5 e 18 anni è stato utilizzato un dosaggio iniziale di fluoxetina di $5-10 \mathrm{mg} /$ die oppure di $10 \mathrm{mg}$ tre volte alla settimana [16]. La dose massima in questi soggetti può essere aggiustata fino ad un massimo di $20 \mathrm{mg} /$ die.

\section{Interazioni farmacologiche}

In letteratura sono riportate le osservazioni cliniche relative alle interazioni della fluoxetina con oltre 100 farmaci. Non tutte le interazioni studiate sono risultate problematiche o rischiose per il paziente.

Nella Tabella 4 riportiamo sinteticamente le interazioni che possono dare conseguenze cliniche importanti e causare reazioni avverse di grado moderato-grave per il paziente e che, a nostro avviso, è necessario conoscere quando si utilizza la fluoxetina. Accanto alle conseguenze note, viene riportata la gravità e vengono indicate alcune norme utili per prevenire o gestire gli eventuali danni.

La maggior parte delle interazioni tra fluoxetina e altri farmaci riconosce un meccanismo farmacocinetico. In particolare, la fluoxetina può interagire a livello degli isoenzimi del citocromo P450 con altri farmaci che utilizzano le stesse vie metaboliche.

La fluoxetina è in grado di inibire l'attività enzimatica dei citocromi CYP2D6, CYP2C19e CYP3A4 e può quindi indurre un iperaccumulo dei farmaci che sono inattivati da tali enzimi.

È noto che gli antidepressivi SSRI differiscono tra loro per la capacità di inibire i diversi isoenzimi del citocromo $\mathrm{P} 450$ ed in particolare il CYP2D6 [17-19].

Studi in vitro indicano il seguente ordine di potenza nell' inibire l'isoenzima CYP2D6: paroxetina $>$ norfluoxetina $>=$ fluoxetina $>$ sertralina $>=$ desmetilsertralina. La fluvoxamina e il citalopram hanno scarsa attività inibitrice sul CYP2D6.

L'importanza clinica dell' azione inibitrice della fluoxetina sull'isoenzima CYP2D6 è stata documentata in un recente lavoro condotto su un gruppo di volontari sani con l'obiettivo di analizzare l'andamento temporale dell'inibizione enzimatica durante e dopo la sospensione del trattamento con alcuni antidepressivi SSRI [20].

Una quarantina di soggetti sono stati randomizzati in tre gruppi, ognuno dei quali è stato trattato rispettivamente con fluoxetina (20mg/die per 28 giorni), paroxetina (20mg/die per 10 giorni) o sertralina (50mg/die per 3 giorni $+100 \mathrm{mg} /$ die per 10 giorni). Durante il trattamento con i tre SSRI l'inibizione dell'attività enzimatica del CYP2D6 aumentò significativamente rispetto ai valori basali: allo steady-state presunto, prima di sospendere il trattamento, l'inibizione era aumentata di 55,3 +/-126,6 


\begin{tabular}{|c|c|c|c|}
\hline Farmaco & Sintomi & Gravità & Considerazioni cliniche \\
\hline Aloperidolo & Tossicità da aloperidolo (pseudoparkinsonismo,atassia) & Moderata & \\
\hline Almotriptan & Debolezza, iperriflessia, incoordinazione & Moderata & \\
\hline $\begin{array}{l}\text { Alprazolam, } \\
\text { diazepam, } \\
\text { triazolam, } \\
\text { zolpidem }\end{array}$ & $\begin{array}{l}\text { Incremento delle concentrazioni ematiche delle benzodiazepine con } \\
\text { conseguente tossicità (sonnolenza, atassia, impaccio psicomotorio, } \\
\text { ipotensione) dovute alla inibizione da parte della fluoxetina del } \\
\text { CYP3A4. }\end{array}$ & Moderata & $\begin{array}{l}\text { Ridurre le dosi di benzodiazepine o } \\
\text { considerare l'uso di molecole quali } \\
\text { lorazepam od oxazepam che sono } \\
\text { maggiormente metabolizzate mediante } \\
\text { glucuronazione }\end{array}$ \\
\hline $\begin{array}{l}\text { Amitriptilina, } \\
\text { desipramina, } \\
\text { imipramina, } \\
\text { nortriptilina }\end{array}$ & $\begin{array}{l}\text { Tossicità da amitriptilina } \\
\text { (secchezza delle fauci, ritenzione urinaria, sedazione) }\end{array}$ & Moderata & $\begin{array}{l}\text { Riduzione del } 50 \%-70 \% \text { la dose dei } \\
\text { triciclici e monitorare il livello plasmatico dei } \\
\text { triciclici }\end{array}$ \\
\hline Bupropione & Aumento delle concentrazioni ematiche di fluoxetina & Moderata & $\begin{array}{l}\text { Considerare l'opportunità dell'associazione } \\
\text { ed eventualmente ridurre il dosaggio di } \\
\text { fluoxetina }\end{array}$ \\
\hline Cannabis & Mania & Moderata & $\begin{array}{l}\text { Sconsigliare l'assunzione concomitante dei } \\
\text { derivati della cannabis }\end{array}$ \\
\hline Carbamazepina & $\begin{array}{l}\text { Tossicità da carbamazepina (atassia, nistagmo, diplopia, cefalea, } \\
\text { vomito, apnea, convulsioni, coma) }\end{array}$ & Moderata & Monitoraggio terapeutico \\
\hline Claritromicina & Delirio e psicosi & Elevata & Evitare l'associazione \\
\hline Clozapina & $\begin{array}{l}\text { Aumentato rischio di tossicità da clozapina (sedazione, convulsioni, } \\
\text { ipotensione) }\end{array}$ & Moderata & \\
\hline Delavirdina & Aumento del $50 \%$ delle concentrazioni di delavirdina & Moderata & Prudenza nell'associazione \\
\hline $\begin{array}{l}\text { Dexfenfluramina, } \\
\text { fenfluramina }\end{array}$ & $\begin{array}{l}\text { Sindrome serotoninica (ipertensione, ipertermia, miocloni, alterazioni } \\
\text { mentali) }\end{array}$ & Elevata & $\begin{array}{l}\text { Non dovrebbero essere usati in } \\
\text { associazione con fluoxetina }\end{array}$ \\
\hline Destrometorfano & $\begin{array}{l}\text { Tossicità da destrometorfano (nausea, vomito, allucinazioni) e } \\
\text { sindrome serotoninica (ipertensione, ipertermia, miocloni, alterazioni } \\
\text { mentali) }\end{array}$ & Elevata & $\begin{array}{l}\text { Prudenza nell'associazione ed eventualmente } \\
\text { riduzione del destrometorfano }\end{array}$ \\
\hline $\begin{array}{l}\text { Digitossina, } \\
\text { digossina }\end{array}$ & Aumentato rischio di tossicità da digitale (nausea, vomito e aritmia) & Moderata & Monitoraggio terapeutico della digitale \\
\hline Diltiazem & $\begin{array}{l}\text { Aumentato rischio di tossicità da diltiazem (nausea, rash cutaneo, } \\
\text { edema, cefalea) }\end{array}$ & Moderata & \\
\hline Droperidolo & $\begin{array}{l}\text { Aumentato rischio di cardiotossicità (allungamento dell'intervallo QT, } \\
\text { torsioni di punta, arresto cardiaco) }\end{array}$ & Elevata & Estrema prudenza nell'associazione \\
\hline $\begin{array}{l}\text { Derivati } \\
\text { ergotaminici }\end{array}$ & $\begin{array}{l}\text { Aumentato rischio di ergotismo (nausea, vomito ischemia } \\
\text { vasospastica) }\end{array}$ & Elevata & $\begin{array}{l}\text { Non dovrebbero essere usati in } \\
\text { associazione con fluoxetina }\end{array}$ \\
\hline Furazolidone & Debolezza, iperriflessia, incoordinazione & $\begin{array}{l}\text { Elevata, in } \\
\text { alcuni casi } \\
\text { fatale }\end{array}$ & Evitare l'associazione \\
\hline Gingko & $\begin{array}{l}\text { Sindrome serotoninica } \\
\text { (ipertensione, ipertermia, miocloni, alterazioni mentali) }\end{array}$ & Moderata & \\
\hline Ketorolac & Allucinazioni & Moderata & Utilizzo di un analgesico alternativo \\
\hline Litio & Possibile tossicità da litio (tremori, confusione, stanchezza) & Moderata & Monitoraggio terapeutico \\
\hline IMAO & $\begin{array}{l}\text { Tossicità a livello del SNC o sindrome serotoninica (ipertensione, } \\
\text { ipertermia, miocloni, alterazioni mentali) }\end{array}$ & Moderata & \\
\hline Fenitoina & $\begin{array}{l}\text { Sindrome serotoninica (ipertensione, ipertermia, miocloni, alterazioni } \\
\text { mentali) }\end{array}$ & Moderata & \\
\hline Pimozide & Bradicardia e sonnolenza & Elevata & $\begin{array}{l}\text { Particolare attenzione ai soggetti anziani e/o } \\
\text { affetti da patologie cardiache }\end{array}$ \\
\hline Ritonavir & Alterazioni del sistema cardiaco e neurologico & Moderata & \\
\hline Sibutramina & $\begin{array}{l}\text { Sindrome serotoninica (ipertensione, ipertermia, miocloni, alterazioni } \\
\text { mentali) }\end{array}$ & Elevata & Evitare l'associazione \\
\hline Sumatriptan & Stanchezza, iperiflessia e incoordinazione & Elevata & Evitare l'associazione \\
\hline Tioridazina & $\begin{array}{l}\text { Aumentato rischio di cardiotossicità (allungamento dell'intervallo QT, } \\
\text { torsioni di punta, arresto cardiaco) }\end{array}$ & Elevata & Evitare l'associazione \\
\hline Tramadolo & $\begin{array}{l}\text { Aumentato rischio di convulsioni e sindrome serotoninica } \\
\text { (ipertensione, ipertermia, miocloni, alterazioni mentali) }\end{array}$ & Elevata & $\begin{array}{l}\text { Evitare l'associazione specialmente in } \\
\text { pazienti con rischio di crisi convulsive. }\end{array}$ \\
\hline Trazodone & $\begin{array}{l}\text { Tossicità da trazodone (sedazione, secchezza delle fauci, ritenzione } \\
\text { urinaria) o sindrome serotoninica (ipertensione, ipertermia, miocloni, } \\
\text { alterazioni mentali) }\end{array}$ & Elevata & Riduzione del dosaggio di trazodone \\
\hline Triptofano & $\begin{array}{l}\text { Sindrome serotoninica (ipertensione, ipertermia, miocloni, alterazioni } \\
\text { mentali) }\end{array}$ & Elevata & $\begin{array}{l}\text { Ridurre i dosaggi dei due farmaci o } \\
\text { sospendere il triptofano }\end{array}$ \\
\hline Warfarin & Aumento del rischio di sanguinamento & Elevata & $\begin{array}{l}\text { Monitorare i parametri ematici di } \\
\text { coagulazione e monitorare clinicamente il } \\
\text { paziente }\end{array}$ \\
\hline
\end{tabular}

Tabella 4

Principali interazioni farmacologiche con la fluoxetina di rilevante interesse clinico, descritte in letteratura 
volte con la fluoxetina, di 8,6 +/- 5,0 volte con la paroxetina e di $15,6+/-22,44$ volte con la sertralina. Data l'elevata variabilità interindividuale, le differenze di inibizione tra $\mathrm{i}$ tre SSRI non sono risultate significative.

Dopo la sospensione del trattamento l'inibizione dell' attività enzimatica del CYP2D6 riprendeva con velocità significativamente differenti in relazione al farmaco usato. Il ritorno ai valori basali avveniva dopo $63,2+/-5,6$ giorni dalla sospensione del trattamento con fluoxetina e il processo di ripresa avveniva con una costante di tempo (emivita) di circa 7 giorni. Dopo trattamento con paroxetina e con sertralina il ritorno ai valori basali avveniva rispettivamente dopo 20,3 +/- 6,4 e 25,0 +/- 11,0 giorni, con una costante di tempo di circa 3 giorni. Durante questo studio nessun soggetto si è trasformato da metabolizzatore rapido a metabolizzatore lento [20].

\section{Interazioni con alimenti ed alcolici}

La presenza di cibo nello stomaco rallenta la velocità di assorbimento della fluoxetina ma non ne riduce la biodisponibilità totale. Pertanto la fluoxetina può essere somministrata indifferentemente in concomitanza o lontano dai pasti [6].

La concomitante somministrazione di fluoxetina e alcol non causa un potenziamento dell'azione psicomotoria dell'alcol né un'inibizione del metabolismo dell'etanolo.

\section{PROFILOTERAPEUTICO}

\section{Efficacia clinica}

L'efficacia clinica della fluoxetina è stata documentata adeguatamente in vari disturbi dell'umore e dell'ansia e oggi le registrazioni del prodotto in Italia, e nella maggior parte dei paesi industrializzati, riconoscono ufficialmente come indicazioni il trattamento della depressione, della bulimia nervosa, del disturbo ossessivo-compulsivo. Nel 2000, la Food and Drug Administration (FDA) degli Stati Uniti ha accettato la domanda di registrazione della fluoxetina per il trattamento dei disturbi disforici premestruali e più recentemente ha registrato il farmaco anche per i pazienti in età pediatrica compresa dai 7 ai 17 anni.

Diverse altre indicazioni della fluoxetina sono state studiate e documentate, soprattutto negli ultimi anni, senza che tuttavia ottenessero un riconoscimento ufficiale dalle autorità regolatorie; sicché il campo delle applicazioni cliniche della fluoxetina è andato continuamente ampliandosi e parallelamente è aumentato l'uso "off-label" dei prodotti contenenti questo principio attivo.
La Figura 1 riporta la distribuzione percentuale delle indicazioni per le quali viene impiegata la fluoxetina dai medici italiani negli ultimi anni. Oltre il $60 \%$ delle prescrizioni riguarda il trattamento di un episodio di depressione, circa il $20 \%$ i disturbi d'ansia e il $6 \%$ i disturbi ossessivi-compulsivi. Oltre il $12 \%$ delle prescrizioni riguarda una molteplicità di altre indicazioni varie, alcune note da tempo, altre documentate più recentemente. Il livello di documentazione dell' efficacia clinica per le indicazioni secondarie non ufficialmente riconosciute dalle registrazioni delle specialità varia molto da caso a caso.

Nella Tabella 5 riportiamo una sintesi delle attuali conoscenze circa l'efficacia della fluoxetina nelle diverse indicazioni testate, come risulta dalla letteratura disponibile.

\section{Depressione maggiore}

L'effetto antidepressivo della fluoxetina nel trattamento dell'episodio depressivo e nel mantenimento è stato ben documentato in passato da diversi trial clinici controllati, alcuni verso placebo.

Negli ultimi anni le proprietà terapeutiche della fluoxetina nella depressione sono state ulteriormente approfondite con ricerche cliniche volte a documentare soprattutto la sua efficacia e tollerabilità comparativamente ad altri SSRI e a nuovi antidepressivi, sia in condizioni controllate sia in assetto naturalistico.

\section{A. Trattamento a breve termine}

L'effetto antidepressivo della fluoxetina è stato studiato in trial clinici controllati con placebo a dosi variabili da 20 a $80 \mathrm{mg} /$ die [21-25].

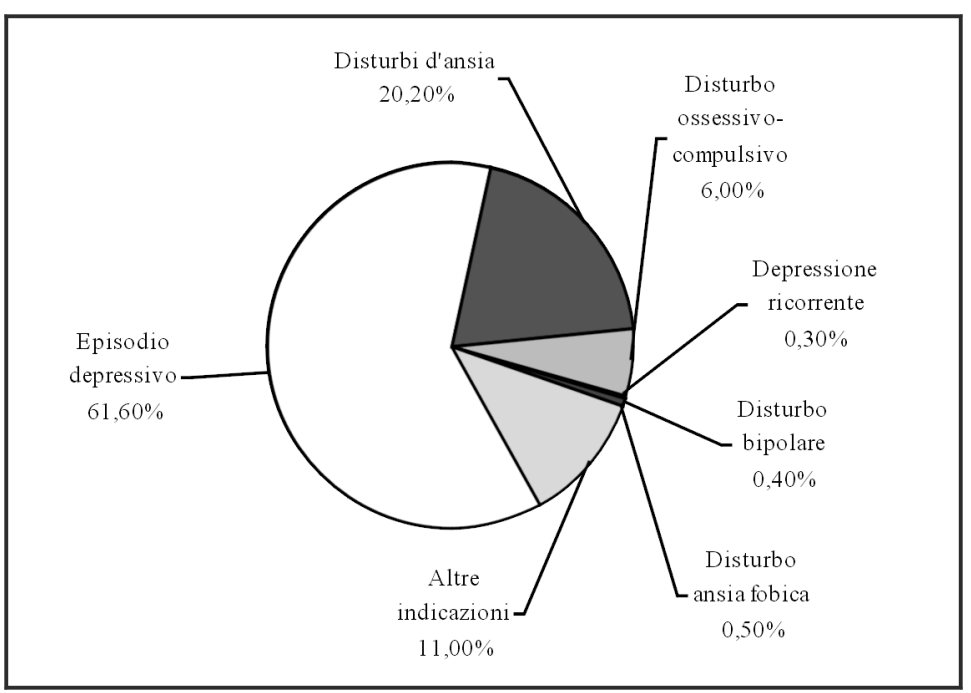

Figura 1

Distribuzione percentuale delle prescrizioni di fluoxetina in Italia in funzione della diagnosi (dati SPM $4^{\circ}$ QTR anno 2001 dicembre-gennaio) 


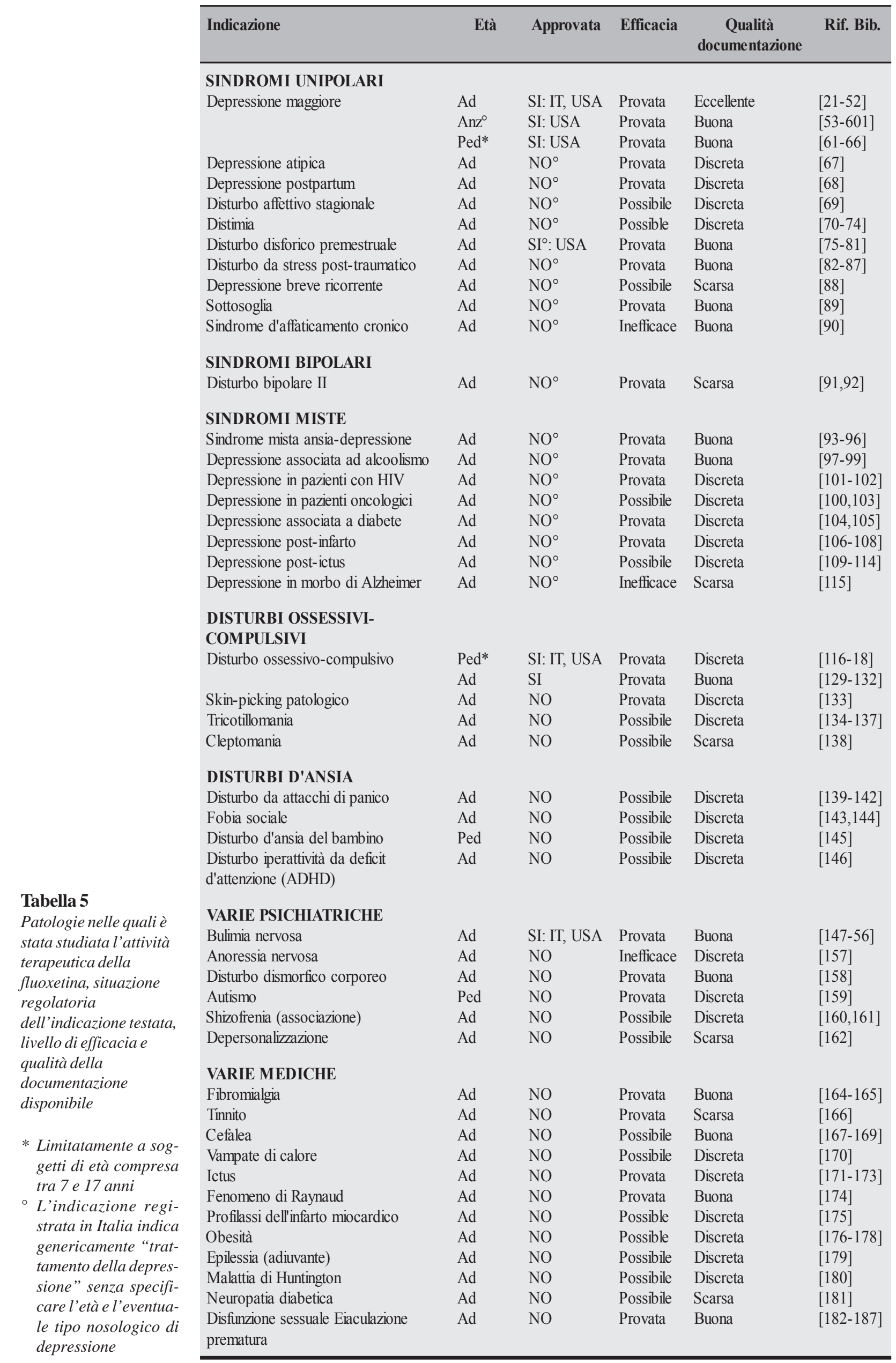


Il dosaggio iniziale per il trattamento della depressione è di $20 \mathrm{mg} /$ die somministrati al mattino. Qualora con questo dosaggio la sintomatologia depressiva non migliori, a distanza di alcune settimane (2-4), è possibile incrementare il dosaggio sino a raggiungere la dose massima di $80 \mathrm{mg} /$ die da somministrare come singola dose mattutina o in dosi refratte. A causa della lunga emivita del farmaco e del suo metabolita attivo, il miglioramento dell'effetto antidepressivo è osservabile solamente dopo alcune settimane dall'aggiustamento posologico.

In un recente lavoro condotto su 182 pazienti ambulatoriali affetti da depressione maggiore e trattati per 8 settimane con fluoxetina alla dose giornaliera di $20 \mathrm{mg}$, la risposta terapeutica positiva (riduzione del $30 \%$ della scala Hamilton-D e successiva riduzione fino al 50\% entro 8 settimane) valutata dopo 2-4 e 6 settimane di trattamento è stata riscontrata rispettivamente nel $55,5 \%, 80,2 \%$ e $89,5 \%$ dei pazienti. La mancanza di risposta terapeutica dopo 4-6 settimane di trattamento è stata associata ad una probabilità del $73-88 \%$ che il paziente non risponda entro 8 settimane [26].

L'efficacia a breve termine della fluoxetina nella depressione maggiore è ritenuta equivalente a quella dei triciclici. Studi meno recenti avevano dimostrato la sostanziale equivalenza della fluoxetina con l'imipramina e l'amitriptilina [9,27,28].

Una recente meta-analisi, attuata analizzando i trial clinici randomizzati disponibili secondo "intention-to-treat", ha esaminato l'efficacia clinica e altri end-point della fluoxetina nel trattamento a breve termine della depressione maggiore, confrontandola con quella del placebo e dei triciclici. L'efficacia della fluoxetina si è confermata significativamente superiore a quella del placebo. Nei trial di confronto con i triciclici, l'efficacia della fluoxetina risultava tendenzialmente superiore se nell' analisi venivano incluse tutte le ricerche o solo quelle condotte negli USA, mentre risultava inferiore se venivano incluse solo quelle condotte in altri paesi non-USA. Includendo nell'analisi tutti i trial, i risultati della meta-analisi hanno dimostrato che una minor percentuale di pazienti trattati con fluoxetina abbandonava il trattamento a causa di eventi avversi [29].

In una diversa meta-analisi, condotta selezionando 315 trial pubblicati tra il 1980 e il 1998, gli autori hanno confermato che i nuovi antidepressivi, compresa la fluoxetina, presentano un' efficacia clinica significativamente superiore al placebo nel trattamento della depressione maggiore e della distimia. Al contrario non è stato possibile evidenziare alcuna significativa differenza tra vecchi e nuovi antidepressivi e tra i diversi nuovi antidepressivi. Questi risultati sono applicabili ad una popo- lazione di pazienti adulti e anziani, mentre al momento della meta-analisi, i dati riguardanti pazienti in età pediatrica erano ancora inconcludenti [30].

La compliance dei pazienti trattati per 12 settimane con fluoxetina dai medici di base è stata valutata in uno studio randomizzato in aperto, in confronto con un triciclico, la dotiepina. La ricerca ha evidenziato che l'uso di fluoxetina è associato ad una più sostenuta compliance nel tempo (curva di sopravvivenza), sebbene gli indicatori crudi di compliance utilizzati non abbiano raggiunto la significatività statistica [31].

Alcune ricerche cliniche controllate più recenti, non incluse nelle meta-analisi citate, hanno confermato la sostanziale equivalenza terapeutica tra fluoxetina, sertralina e paroxetina, $i$ tre SSRI più utilizzati, nel trattamento dell'episodio depressivo [32-34]. In tutti tre i lavori sono stati studiati pazienti ambulatoriali. Lo studio più recente di Fava e collaboratori, oltre ad aver confermato l'equivalenza terapeutica dei parametri di efficacia e tollerabilità di fluoxetina, sertralina e paroxetina nel trattamento acuto della depressione maggiore, ha anche dimostrato un'equivalente attività nel migliorare l'insonnia associata alla depressione, indipendentemente dai livelli basali insonnia [34].

Una forma particolare di equivalenza tra fluoxetina, sertralina e paroxetina è stata evidenziata in uno studio che aveva come obiettivo principale definire la probabilità che un paziente trattato inizialmente con un SSRI termini il trattamento a breve termine con lo stesso antidepressivo e di stimare, quindi, se ad ognuno degli SSRI scelto inizialmente sia associata una differente frequenza di cambio.

La ricerca ha dimostrato che circa il $25 \%$ dei pazienti trattati inizialmente con fluoxetina, sertralina o paroxetina passavano ad un altro antidepressivo nel corso del loro trattamento. Sotto questo aspetto, dunque, i tre antidepressivi risultavano equivalenti come efficacia media sulla popolazione.

Tuttavia, a livello del singolo paziente i tre SSRI testati non erano equivalenti perché soggetti che sospendevano il farmaco iniziale per mancanza di efficacia o per scarsa tollerabilità generalmente rispondevano in modo adeguato ad uno degli altri due antidepressivi [35].

D'altra parte, in un precedente trial, la fluoxetina somministrata dosi variabili da 20 a $60 \mathrm{mg} /$ die è risultata efficace nel 63\% (67/106) dei pazienti che non avevano risposto all'iniziale terapia con sertralina [36].

\section{B. Terapia di mantenimento}

La terapia di mantenimento è necessaria per prevenire nuovi episodi nei casi recidivanti. 
Pazienti affetti da disturbo depressivo maggiore rimangono a rischio di recidive dopo la remissione dei sintomi e spesso continuano a denunciare la presenza di sintomi attenuati o sottosoglia.

Uno studio clinico controllato, condotto su oltre 839 pazienti affetti da depressione maggiore che avevano risposto positivamente al trattamento iniziale di 12 settimane con fluoxetina $20 \mathrm{mg} / \mathrm{die}$, ha dimostrato che sono necessarie almeno altre 26 settimane (sei mesi) di trattamento continuativo per ottenere un buon controllo sulle recidive. Lo studio, tuttavia, non ha chiarito se un trattamento di durata superiore alle 38 settimane possa produrre ulteriori benefici nel prevenire gli episodi recidivanti di depressione [37].

Uno studio più recente, condotto in Spagna, ha verificato l'efficacia della fluoxetina $20 \mathrm{mg} /$ die nella terapia di mantenimento protratta per 48 settimane. Pazienti affetti da depressione unipolare trattati con successo con fluoxetina $20 \mathrm{mg} /$ die nella fase iniziale di 32 settimane, sono stati randomizzati in due gruppi e trattati in doppio cieco rispettivamente con fluoxetina $20 \mathrm{mg} /$ die o con placebo. Nel gruppo trattato con fluoxetina la percentuale di soggetti che hanno avuto una recidiva era significativamente inferiore a quella del gruppo placebo $(20 \%$ vs $40 \%, \mathrm{p}=0,01)$ e l'intervallo libero da sintomi, stimato con le curve di sopravvivenza (Kaplan-Meyer) era significativamente più lungo (295 giorni vs 192 giorni, $\mathrm{p}=0,002$ ). Il trattamento a lungo termine con fluoxetina è stato ben tollerato e nessun paziente ha abbandonato la terapia a causa di reazioni avverse; nel gruppo trattato con placebo è stato registrato un numero significativamente superiore di reazioni ansiose e due pazienti hanno abbandonato il trattamento a causa di reazioni avverse [38].

Alcuni autori, considerando la notevole lunghezza dell'emivita della fluoxetina e soprattutto del suo metabolita attivo, hanno voluto provare l'efficacia di una posologia di mantenimento basata sulla somministrazione di una dose settimanale di $60 \mathrm{mg}$. Nella fase iniziale i soggetti, affetti da depressione maggiore, erano stati trattati in aperto con fluoxetina alla dose abituale di $20 \mathrm{mg} /$ die per 7 settimane. Successivamente, soggetti che avevano raggiunto uno score della scala di Hamilton-D $<=12$ sono stati randomizzati in tre gruppi e trattati in doppio cieco per altre 7 settimane rispettivamente con fluoxetina $20 \mathrm{mg} / \mathrm{die}, 60 \mathrm{mg} / \mathrm{setti}-$ mana o placebo. I gruppi erano formati da pochi pazienti e durante la fase di mantenimento non è stato possibile rilevare differenze statisticamente significative tra gruppi nei valori medi della scala di Hamilton-D e nelle frequenze di drop-out dei pazienti. Tuttavia i gruppi trattati con fluoxetina dimostravano una tendenziale riduzione dei sintomi depressivi, rispetto al placebo. L'assenza di differenza tra gruppi trattati e placebo è stata giustificata invocando un effetto carry-over dalla precedente fase di trattamento, dovuto alla lentezza dell'eliminazione della norfluoxetina. Le concentrazioni plasmatiche di norfluoxetina nel gruppo trattato con la dose di $60 \mathrm{mg} / \mathrm{settimana}$ erano inferiori di circa il $50 \%$ rispetto al gruppo trattato con la dose abituale di $20 \mathrm{mg} / \mathrm{die}[39,40]$.

Questo studio preliminare indica la possibilità di una posologia settimanale nella fase di mantenimento. Tuttavia, è necessario confermare l'efficacia di tale schema posologico su una casistica più ampia e analizzando la prevenzione delle recidive per tempi più lunghi di mantenimento, almeno sei mesi.

L'affiancamento di una psicoterapia cognitiva al trattamento farmacologico è ritenuto generalmente utile. Inoltre, la psicoterapia sarebbe in grado di prevenire le recidive anche dopo la sospensione del trattamento farmacologico [41-43]. Un recente studio, tuttavia, non ha rilevato differenze statisticamente significative nelle frequenza di recidive e di drop-outs tra un gruppo trattato per 28 settimane con fluoxetina alla dose di 40mg/die e un gruppo parallelo trattato con psicoterapia cognitiva in aggiunta alla stessa dose di farmaco [44]. La mancata differenza è probabilmente dovuta all'incremento della dose che rende inutile o maschera l'effetto additivo della psicoterapia.

Fattori psicosociali, condizioni di stress, difficoltà di coppia sono tra le principali cause correlabili alle recidive. Lo sviluppo di sintomi subsindromici durante la fase di mantenimento è predittivo di una maggior probabilità di recidiva della depressione dopo la sospensione del trattamento $[45,46]$.

La depressione è una patologia molto diffusa che spesso viene diagnosticata e trattata direttamente dal medico di medicina generale. La fluoxetina per il suo profilo di efficacia e tollerabilità può essere prescritta con sicurezza anche dal medico di famiglia, oltre che dallo specialista. D'altra parte l'efficacia del farmaco è stata studiata prevalentemente su campioni molto selezionati di pazienti, gestiti da centri ospedalieri o ambulatoriali specialistici. L'efficacia e la tollerabilità del farmaco in pazienti trattati dal medico di medicina generale e gestiti in condizioni naturalistiche sono state valutate solo recentemente dallo studio ARTIST [47]. In questa ampia ricerca multicentrica, condotta su 573 pazienti adulti affetti da depressione e gestiti direttamente dai medici di medicina generale, l'efficacia e la tollerabilità della fluoxetina sono state confrontate con quelle della sertralina e della paroxetina. Il trattamento 
previsto era di nove mesi e come outcomes sono stati valutati, oltre ai sintomi della depressione, diversi parametri correlabili alla qualità di vita: capacità lavorativa, integrazione sociale, funzionalità fisica, vitalità, capacità di concentrazione e di memorizzazione, dolorabilità, sonno, funzionalità sessuale. I pazienti sono stati randomizzati ai tre gruppi di trattamento e i medici erano liberi di cambiare l'antidepressivo se quello inizialmente assegnato non risultava efficace o ben tollerato. Fluoxetina, sertralina e paroxetina, utilizzati in condizioni naturali dal medico di medicina generale in trattamenti prolungati di 9 mesi, hanno evidenziato equivalenti efficacia e tollerabilità cliniche ed equivalenti utilità nel migliorare le diverse dimensioni valutate della qualità di vita dei pazienti, ad ognuno di tempi di follow-up previsti dal protocollo (1, 3, 6 e 9 mesi).

Questo studio ha definitivamente confermato l'efficacia e l'utilità di un trattamento antidepressivo prolungato con un SSRI in una popolazione di pazienti trattati in modo naturalistico ed ha definitivamente evidenziato l'equivalenza della fluoxetina con la sertralina e la paroxetina.

\section{Terapia di associazione}

Il trattamento polifarmacologico può essere necessario per i pazienti depressi che non hanno una remissione completa della sintomatologia.

Alcuni lavori hanno dimostrato un potenziamento dell'efficacia antidepressiva della fluoxetina dovuta all'associazione con altri farmaci.

Indagini pilota hanno dimostrato che una certa percentuale di pazienti depressi, che non hanno risposto o che hanno risposto solo parzialmente al trattamento iniziale con fluoxetina $20 \mathrm{mg} /$ die, può avere beneficio da un aumento della dose a 40-60mg/die o dall' associazione con litio (300-600mg/die) o con desipramina (25-50mg/die) [48]. Questo risultato è stato confermato recentemente da uno studio allargato, condotto su oltre 100 pazienti affetti da depressione maggiore resistenti o parzialmente responders al trattamento con fluoxetina $20 \mathrm{mg} /$ die per 8 settimane. Questi pazienti sono stati randomizzati a tre differenti strategie di trattamento in doppio cieco per altre 4 settimane, consistenti rispettivamente nella dose di fluoxetina di 40-60mg/die, dell' associazione con litio (300-600mg/die) o nell' associazione con desipramina (25-50mg/die). La percentuale di responders, di responders parziali e di nonresponders non è risultata significativamente differente nei tre gruppi di trattamento, così come non è risultata differente la frequenza di drop-outs. Sebbene non statisticamente signi- ficativa, l'efficacia di dosi più elevate di fluoxetina era tendenzialmente superiore a quella delle due associazioni [49].

Due diversi lavori, condotti in doppio cieco versus placebo su piccoli gruppi di pazienti depressi, hanno documentato un incremento di efficacia antidepressiva dell'associazione della fluoxetina con il clonazepam o con pindololo [50,51].

L'associazione fluoxetina $20 \mathrm{mg} / \mathrm{die}+$ pindololo $7,5 \mathrm{mg} /$ die ha prodotto un miglioramento significativo dell' effetto antidepressivo sia come percentuale di pazienti migliorati dopo 6 settimane di trattamento sia come riduzione della scala di Hamilton-D. Il vantaggio di tale associazione può essere attribuito all' azione di contrasto del pindololo sulla riduzione dell'attività neuronale serotoninergica causata dai farmaci SSRI, con conseguente potenziamento del loro effetto terapeutico [51].

Una recente ricerca controllata con placebo ha dimostrato un significativo potenziamento dell'azione antidepressiva della fluoxetina $20 \mathrm{mg} /$ die indotta dalla supplementazione giornaliera di 500 microgrammi di acido folico. La somministrazione di acido folico ha indotto un incremento dei folati e una riduzione dei livelli plasmatici di omocisteina, ma la diminuzione di omocisteina è risultata statisticamente significativa solo nelle donne. Gli uomini necessitano di dosi più elevate di acido folico per ridurre i livelli di omocisteina. Il miglioramento dei punteggi della depressione si è verificato nel 94\% delle donne che hanno ricevuto la supplementazione di acido folico contro il $61 \%$ che hanno avuto il placebo [52].

\section{Depressione nell'anziano}

Diversi lavori hanno dimostrato l'efficacia e l'elevata sicurezza della fluoxetina nel trattamento della depressione maggiore di soggetti anziani [53-59].

La valutazione dell' effetto antidepressivo in una popolazione di anziani è complicata dal fatto che le frequenti malattie organiche cronico-degenerative che affliggono l'anziano alterano la qualità della vita e possono avere un impatto variabile sullo stato funzionale del soggetto e sugli esiti del trattamento antidepressivo. La presenza e il numero di malattie croniche non influenza il trattamento antidepressivo, ma la presenza nell' anamnesi di una pregressa malattia organica è stata associata ad una maggiore risposta alla fluoxetina e ad una minore risposta placebo [53].

Lo studio condotto in doppio cieco versus placebo su un gruppo di anziani ricoverati e trattati per 8 settimane con placebo ha dimostrato che la risposta al farmaco era significativamente superiore al placebo nei soggetti che 
avevano una grave malattia che metteva in pericolo la loro vita, o avevano una malattia cerebrovascolare, ma non erano dementi, non assumevano analgesici o erano in trattamento con analgesici più potenti del paracetamolo [54].

A differenza del placebo, la fluoxetina induce una risposta persistente a partire dalla quarta settimana di trattamento. La presenza di sonnolenza prima dell'inizio del trattamento è stata associata ad una precoce risposta sia al farmaco sia al placebo e può quindi essere considerata un marker dell'effetto placebo. La presenza di cefalea prima e dopo la randomizzazione è stata associata ad una buona risposta alla fluoxetina, mentre la presenza di ansia è stata associata ad una cattiva risposta [56].

In due differenti trial clinici condotti in doppio cieco l'efficacia e la tollerabilità della fluoxetina sono risultate equivalenti rispettivamente a quella della sertralina e della paroxetina $[58,59]$. Il trattamento prolungato fino ad 1 anno non ha comportato deterioramento delle funzioni cognitive, anzi le ha migliorate.

Rispetto alla sertralina, il trattamento per 12 settimane con fluoxetina ha comportato una maggiore perdita di peso [55].

L'accettazione della terapia antidepressiva è stata molto scarsa tra un gruppo di pazienti anziani depressi affetti da broncopneumopatia cronico-ostruttiva (BPCO) probabilmente per un'eccessiva apprensione di questa categoria di pazienti. I casi di BPCO depressi non trattati si sono cronicizzati [60].

\section{E. Depressione dell'età pediatrica}

La fluoxetina è efficace anche nel trattamento della depressione maggiore dei bambini e degli adolescenti. Due studi condotti in doppio cieco hanno dimostrato che la fluoxetina, alla dose di $20 \mathrm{mg} / \mathrm{die}$ (con un' eventuale fase iniziale a $10 \mathrm{mg} / \mathrm{die}$ ), è significativamente superiore al placebo nel controllare i sintomi della depressione maggiore durante il trattamento acuto in un gruppo di soggetti di 7-17 anni. Nel gruppo fluoxetina e nel gruppo placebo la percentuale di successi è stata rispettivamente del $56 \%$ e del $33 \%$. La remissione completa dei sintomi, tuttavia, è stata inferiore $(31 \%$ vs $23 \%)[61,62]$. Non è stato possibile evidenziare un fattore predittivo della risposta clinica [63]. La fluoxetina è stata ben tollerata.[62]

Alcune altre ricerche condotte in aperto e in condizioni naturalistiche hanno confermato l'efficacia e l'utilità della fluoxetina nel trattamento della depressione del bambino e dell'adolescente $[64,65]$.

La fluoxetina sembra essere utile anche negli adolescenti depressi con tendenza all'abuso di alcolici [66].

\section{Depressione atipica}

Una recente ricerca clinica controllata ha dimostrato che la fluoxetina è efficace nel trattamento della depressione atipica secondo DSM-IV, un sottogruppo della depressione maggiore. Il trattamento in doppio cieco con fluoxetina per 10 settimane è risultato efficace quanto l'imipramina: entrambi gli antidepressivi erano significativamente superiori al placebo. La tollerabilità della fluoxetina era migliore di quella del triciclico [67].

\section{Distimia}

Un numero limitato di studi clinici, la maggior parte dei quali condotti in aperto, supporta l'ipotesi che la fluoxetina sia efficace nella distimia primaria dell'adulto e dell'anziano. Tuttavia, ulteriori studi controllati e a lungo termine sono necessari per stabilire con sicurezza l'efficacia della fluoxetina nella distimia.

In uno studio in aperto, la fluoxetina alla dose variabile di $20-60 \mathrm{mg} /$ die è risultata efficace in oltre il $70 \%$ dei pazienti, con una migliore risposta nei soggetti che presentavano distimia subaffettiva [70].

In un' altra ricerca l'efficacia della fluoxetina nella distimia dopo 16 settimane di trattamento è stata solo parziale [71].

In uno dei pochi studi controllati, randomizzati, condotti in doppio cieco versus placebo su un numero relativamente elevato $(\mathrm{n}=140)$ di pazienti affetti da distimia primaria, la fluoxetina $20 \mathrm{mg} /$ die ha indotto un miglioramento significativo dei parametri clinici e funzionali entro 3 mesi di trattamento. I soggetti non responders alla dose di $20 \mathrm{mg} / \mathrm{die}$ hanno avuto qualche miglioramento con un incremento della posologia. Dopo sei mesi di trattamento i soggetti che inizialmente avevano risposto positivamente risultarono ulteriormente migliorati e il 50\% dei non-responders è risultato migliorato con l' aumento delle dosi. La qualità e frequenze di reazioni avverse registrate nel gruppo trattato con fluoxetina non furono significativamente differenti da quelle del gruppo placebo [72].

Uno studio condotto su un piccolo gruppo di anziani $(n=20)$ affetti da distimia, la somministrazione di fluoxetina alla dose media di $35,5 \mathrm{mg} /$ die è risultata efficace nel $60 \%$ dei soggetti [73].

Alcuni dati preliminari fanno ipotizzare che la fluoxetina possa essere utile anche nel trattamento della distimia dell'età pediatrica. Un piccolo gruppo di bambini o adolescenti, affetti da distimia o da depressione doppia, che non avevano risposto positivamente ad una terapia psicosociale sono stati trattati per 8 settimane con fluoxetina $20 \mathrm{mg} / \mathrm{die}$ : il $73 \%$ di 
questi pazienti ha fatto registrare una normalizzazione dei sintomi al termine della terapia farmacologia [74].

\section{Disturbi depressivi non altrimenti specificati (NAS)}

Ricerche cliniche controllate indicano che la fluoxetina è efficace anche in alcuni disturbi depressivi che non rientrano nella classificazione delle depressioni maggiori o della distimia, come il disturbo disforico premestruale, il disturbo da stress post-traumatico, la depressione ad episodi brevi ricorrenti e la depressione sottosoglia.

\section{A. Disturbo disforico premestruale}

Alcune ricerche cliniche controllate, attuate con differenti disegni sperimentali, hanno concordemente evidenziato la buona efficacia della fluoxetina nel controllare i sintomi del disturbo disforico premestruale [75-77]. Recenti rassegne hanno sintetizzato i risultati di questi lavori [78,79].

Nelle pazienti con disturbi disforici premestruali la dose di fluoxetina consigliata è di $20 \mathrm{mg} / \mathrm{die}$, somministrata ininterrottamente o in maniera ciclica dal $14^{\circ}$ giorno alla fine del ciclo mestruale. Incrementi di dosaggi non sembrano migliorare il controllo della sintomatologia disforica.

La lunga emivita della fluoxetina e la breve durata dei sintomi porta ad ipotizzare l'utilità di trattamenti ciclici di breve durata nella settimana precedente le mestruazioni. Sono tuttavia necessari ulteriori trial clinici controllati su ampie casistiche.

In uno studio naturalistico di 6 mesi, l' efficacia della fluoxetina è stata confrontata con quella della psicoterapia cognitiva e con l'associazione fluoxetina-psicoterapia. Al termine dei sei mesi il miglioramento dei sintomi e la percentuale di successi non era significativamente differente nei tre gruppi di trattamento. Il gruppo trattato con fluoxetina, tuttavia, ha fatto registrare una più rapida risoluzione dei sintomi, mentre il gruppo trattato con psicoterapia ha manifestato una minor tendenza alla recidiva durante la fase di mantenimento. L'associazione tra fluoxetina e psicoterapia non ha comportato un potenziamento dei risultati [80].

Il trattamento con fluoxetina migliora significativamente la capacità lavorativa delle donne affette da disturbo disforico premestruale [81].

\section{B. Disturbo da stress post-traumatico}

Alcuni lavori clinici controllati, condotti negli ultimi anni, hanno dimostrato una buona efficacia della fluoxetina, significativamente superiore al placebo, nel trattamento del disturbo da stress post-traumatico in gruppi di civili e di militari [82-87].

Per ottenere un effetto clinicamente soddisfacente è necessario utilizzare dosi elevate di fluoxetina $(60 \mathrm{mg} / \mathrm{die})$. Il trattamento acuto con fluoxetina riduce e controlla i sintomi depressivi, migliora la qualità di vita dei pazienti, normalizzandone i parametri di funzionalità fisica, l'attività sociale, la vitalità e lo stato mentale. Il trattamento prolungato è efficace nel prevenire le recidive dei sintomi.

Uno studio preliminare ha documentato che la fluoxetina normalizza la variabilità della frequenza cardiaca, che risulta alterata nel disturbo da stress post-traumatico [86].

Il trattamento con fluoxetina è ben tollerato in questa categoria di pazienti [87].

\section{Depressione breve ricorrente e depressione sottosoglia}

Uno studio preliminare condotto in aperto evidenzia l'efficacia della fluoxetina nei pazienti affetti da depressione ad episodi brevi ricorrenti. Questo risultato dovrà essere convalidato da ulteriori studi controllati in doppio cieco [88].

Una recente rassegna dei lavori clinici di confronto tra fluoxetina e un estratto standardizzato di iperico indica una sostanziale equivalenza terapeutica tra $i$ due trattamenti in pazienti affetti da depressione sottosoglia [89].

\section{Disturbo bipolare II}

Il disturbo bipolare II, presente secondo alcune casistiche in circa il $45 \%$ dei pazienti affetti da depressione maggiore, viene di preferenza trattato con stabilizzatori dell'umore. Analizzando retrospettivamente i risultati clinici di trial di efficacia della fluoxetina a breve e lungo termine, è stato possibile confrontare l'efficacia della fluoxetina nel sottogruppo di pazienti affetti da disturbo bipolare II con quella dei pazienti affetti da depressione unipolare. Durante la fase acuta iniziale l'efficacia della fluoxetina e la frequenza di drop-outs non sono risultati statisticamente differenti nei due sottogruppi. Durante il trattamento di mantenimento la frequenza di recidive è risultata simile nei pazienti bipolari e nei pazienti unipolari. Durante il trattamento iniziale una percentuale lievemente superiore di pazienti bipolari ha presentato un viraggio alla fase maniacale [91].

Per ridurre il rischio di viraggio alla fase maniacale nei pazienti bipolari, alcuni autori hanno suggerito di utilizzare la fluoxetina alla dose di $10 \mathrm{mg}$ due volte alla settimana, come terapia aggiuntiva a stabilizzatori dell'umore [92]. 


\section{Depressione e comorbilità psichiatriche}

\section{A. Disturbo misto ansia-depressione}

Il disturbo misto ansia-depressione, o depressione maggiore con comorbilità del disturbo d'ansia, è un sottotipo molto comune di depressione associato ad un maggior livello di menomazione psicosociale e ad una minor sensibilità al trattamento con farmaci antidepressivi. La fluoxetina e gli altri SSRI sono considerati efficaci anche in questa forma di depressione.

Alcune recenti ricerche condotte in aperto su gruppi di pazienti ambulatoriali hanno confermato l'efficacia della fluoxetina, somministrata per 8 settimane alla dose di $20 \mathrm{mg} / \mathrm{die}$, nel ridurre sia i sintomi della depressione sia i sintomi dell'ansia, comprese eventuali somatizzazioni [93,94].

Uno studio condotto da un gruppo di ricercatori italiani per la prima volta ha studiato l'efficacia della fluoxetina $20 \mathrm{mg} / \mathrm{die}$, come monoterapia, nel trattamento del primo episodio di depressione ansiosa. Lo studio, condotto in aperto, ha confrontato i punteggi delle scale della depressione e dell' ansia misurati prima del trattamento, durante e al termine dei 50 giorni di terapia. I sintomi della depressione e dell' ansia sono significativamente migliorati già dopo 10 giorni e la valutazione soggettiva dell'ansia dopo 20 giorni di trattamento. Al termine del trattamento il miglioramento dei sintomi era complessivamente superiore nel sottogruppo di pazienti affetti da disturbo misto rispetto al sottogruppo di depressi puri. Questa ricerca indica, dunque, che la fluoxetina è in grado di controllare rapidamente i sintomi del disturbo misto ansia-depressione che si manifesti per la prima volta [94].

In una ricerca controllata in doppio cieco la fluoxetina $20 \mathrm{mg} /$ die per 8 settimane è risultata efficace nella depressione mista ansiosodepressiva quanto l'amitriptilina alla dose di 50-250mg/die. Gli effetti collaterali e le reazioni avverse sono state meno frequenti e meno gravi nei soggetti trattati con fluoxetina [95].

Una ricerca clinica condotta in doppio cieco su tre gruppi paralleli randomizzati ha dimostrato l'equivalenza terapeutica tra fluoxetina, sertralina e paroxetina nel controllare i sintomi della depressione e dell' ansia di pazienti affetti da depressione mista ansioso-depressiva. Tutti i pazienti manifestavano un elevato punteggio dei sintomi dell' ansia prima del trattamento. I tre SSRI testati hanno evidenziato una sostanziale equivalenza nel migliorare i punteggi dei sintomi della depressione e dell' ansia, ma la fluoxetina e la sertralina hanno controllato più rapidamente i sintomi dell'ansia durante la prima settimana di trattamento. Le percentuali di successi, miglioramenti e insuccessi e di reazioni avverse non sono risultate significativamente differenti nei tre gruppi di trattamento. Pertanto si può concludere che la fluoxetina presenta un profilo di efficacia e di tollerabilità equivalente a quello della sertralina e della paroxetina nel trattamento acuto dei pazienti affetti da depressione maggiore con elevati livelli di ansia [96].

\section{B. Depressione in alcolisti}

Alcuni trial clinici controllati hanno documentato l'efficacia clinica della fluoxetina nei pazienti depressi alcolisti cronici. Il trattamento acuto e a lungo termine con fluoxetina, oltre a controllare i sintomi della depressione, è risultato utile anche per ridurre il consumo di alcolici e diminuire il grado di dipendenza [97-99].

Nessuna utilità della fluoxetina è stata osservata invece in un gruppo di alcolisti che contemporaneamente abusavano anche di cocaina [98].

\section{Depressione e comorbilità organiche}

Diverse ricerche cliniche hanno documentato l'efficacia e la tollerabilità della fluoxetina nel trattamento di pazienti affetti da depressione con comorbilità organiche.

Una recente rassegna ha evidenziato che la fluoxetina, studiata in trials clinici controllati in doppio cieco, è significativamente superiore al placebo nel trattamento della depressione che si manifesta nei pazienti affetti da infezione HIV/AIDS, da diabete mellito o da ictus, mentre non è superiore al placebo nel ridurre $i$ sintomi depressivi dei pazienti oncologici. L'efficacia della fluoxetina è risultata equivalente a quella della desipramina nell' ictus, nel diabete mellito e nell'HIV/AIDS, ed equivalente a quella della sertralina e della paroxetina nell'HIV/ AIDS [100].

Un recente lavoro ha rivalutato l'efficacia della fluoxetina nel controllare i sintomi della depressione dei pazienti oncologici. In un gruppo di 163 pazienti randomizzati a ricevere in doppio cieco un trattamento antidepressivo con fluoxetina $20 \mathrm{mg} /$ die o placebo per un periodo di 12 settimane, la fluoxetina ha migliorato significativamente i sintomi della depressione e la qualità di vita, risultando al contempo ben tollerata [103].

Nei pazienti diabetici depressi, l'uso di fluoxetina, oltre a ridurre significativamente i sintomi della depressione, è stato correlato con un miglior controllo metabolico della glicemia [105].

La fluoxetina è efficace e sicura nei pazienti cardiopatici e nella depressione post-infarto. Le reazioni avverse della fluoxetina a livello del sistema cardiovascolare sono trascurabili, 
per lo più non differenziabili da quelle del placebo e nettamente inferiori a quelle correlate all'uso di triciclici [106-108].

Diversi trial clinici controllati hanno dimostrato che i trattamenti acuto e prolungato con fluoxetina sono efficaci nel controllare e prevenire la sintomatologia depressiva nei pazienti che hanno subito un ictus cerebrale. Il trattamento è utile anche per facilitare la ripresa funzionale e migliorare l'esito della riabilitazione [109-114].

La fluoxetina non è risultata efficace nei pazienti depressi affetti da morbo di Alzheimer [115].

\section{Disturbo ossessivo-compulsivo}

La fluoxetina è considerata un farmaco di prima scelta per la terapia farmacologica del disturbo ossessivo-compulsivo. La sua efficacia è sostanzialmente equivalente a quella della clomipramina, sebbene alcune rassegne e una meta-analisi propendano per accreditare una lieve superiorità della clomipramina nel controllare i sintomi ossessivi [120-122]. La fluoxetina è in generale meglio tollerata della clomipramina e questo fattore è l'elemento decisivo che tende a condizionare la scelta del trattamento iniziale. Non esiste, tuttavia un consensus su quale debba essere il farmaco di prima scelta nella terapia del disturbo ossessivo-compulsivo.

La dose efficace di fluoxetina nel disturbo ossessivo-compulsivo varia da $20 \mathrm{ad} 80 \mathrm{mg} /$ die [123]. La strategia oggi preferita è quella di iniziare il trattamento con dosi di 20mg/die e di incrementare poi gradualmente la dose fino a raggiungere eventualmente la dose massima di $80 \mathrm{mg} /$ die: così facendo si ottengono buoni risultati terapeutici riducendo il rischio di reazioni avverse [121].

In studi controllati versus placebo la fluoxetina è risultata più efficace del placebo nel migliorare tutti gli indicatori misurati, in particolare i sintomi ossessivi, compulsivi e depressivi. La dose di $60 \mathrm{mg} /$ die è stata associata ad una maggiore riduzione dei punteggi delle scale ossessive-compulsive, rispetto alla dose iniziale di $20 \mathrm{mg} / \mathrm{die}$. La percentuale di successi e il grado di miglioramento dei sintomi sono stati maggiori nei pazienti che avevano una storia di remissione dei sintomi, che non avevano ricevuto un precedente trattamento farmacologico o che presentavano una maggiore gravità del disturbo, specialmente nella componente ossessiva [124].

La terapia con fluoxetina non modifica il ritmo circadiano dei sintomi, né il ritmo circadiano della temperatura corporea, del cortisolo e della melatonina. L'efficacia della fluoxetina non è pertanto correlabile con questi parametri [125].
Non è stato ancora stabilito quale sia la durata ottimale del trattamento, ma per la maggior parte dei pazienti è necessario attuare una terapia a lungo termine. Alcuni studi condotti su piccoli gruppi hanno evidenziato un'elevata percentuale di recidive, variabile dal $65 \%$ al $90 \%$, dopo la sospensione del trattamento farmacologico [126].

Un più recente lavoro ha studiato l'efficacia della fluoxetina a tre livelli dose (20, 40 e 60 $\mathrm{mg} /$ die) nel prevenire le recidive del disturbo ossessivo-compulsivo. I soggetti che avevano risposto al trattamento al termine della fase iniziale di 20 settimane, sono stati randomizzati (in doppio cieco) a proseguire il trattamento con fluoxetina per un altro anno oppure a passare al placebo. Una percentuale significativamente inferiore di soggetti trattati a lungo termine con la dose di $60 \mathrm{mg} /$ die è andata incontro a recidiva, rispetto al placebo $(17,5 \%$ vs $38,0 \%$ ), mentre le dosi di 20 e $40 \mathrm{mg} /$ die hanno fatto registrare una percentuale di recidive non significativamente differente dal placebo [127].

Il trattamento delle recidive può essere fatto con un altro antidepressivo o con lo stesso farmaco utilizzato nel precedente trattamento. Un recente lavoro ha dimostrato che i soggetti che hanno una recidiva dopo la sospensione del trattamento rispondono ancora allo stesso farmaco e alla stessa dose, ma con una minore intensità rispetto al precedente trattamento acuto [128].

Sono considerati refrattari i soggetti che non rispondono positivamente entro 10-12 settimane di trattamento alla massima dose di fluoxetina e/o alla psicoterapia cognitiva. I soggetti non-responders alla fluoxetina nel $20 \%$ dei casi possono essere sensibili ad un altro SSRI o alla clomipramina; la rimanente quota dell' $80 \%$ dovrà invece essere trattata con una terapia di associazione, in genere con aloperidolo o con clonazepam [126].

Recenti ricerche cliniche controllate hanno dimostrato che la fluoxetina alle dosi di 20$60 \mathrm{mg} /$ die è efficace e ben tollerata anche nella popolazione pediatrica (7-17 anni) affetta da disturbo ossessivo-compulsivo [131,132].

\section{Disturbi d'ansia, attacchi di panico e fobia sociale}

Uno studio controllato, condotto in doppio cieco su 243 pazienti affetti da disturbo panico, ha dimostrato che la fluoxetina, somministrata per 10 settimane alla dose di 10 o 20 $\mathrm{mg} /$ die con eventuale mantenimento per altre 24 settimane, ha un' efficacia significativamente superiore al placebo nel ridurre i punteggi delle scale di valutazione delle fobie, dell'evitamento fobico, dell' ansia e dell' ansia 
anticipatoria, della depressione e della funzionalità generale. Il numero totale di attacchi di panico è risultato significativamente ridotto solo nel gruppo trattato con la dose di $10 \mathrm{mg} /$ die. La frequenza e qualità degli eventi avversi e il numero di abbandoni non è stato differente nei tre gruppi. La fluoxetina è, dunque, ben tollerata anche dai pazienti che soffrono di disturbo panico [140].

L'efficacia della fluoxetina è stata confermata anche nella fase di trattamento prolungato di 24 settimane [141].

Uno studio condotto su un piccolo gruppo di pazienti affetti da attacchi di panico ha evidenziato la possibilità di utilizzare una singola dose settimanale di fluoxetina variabile da 10 a $60 \mathrm{mg}$ invece della dose giornaliera. Questo tipo di posologia dovrà essere ulteriormente studiato [142].

La fluoxetina, alla dose variabile da 10 a 60 $\mathrm{mg} / \mathrm{die}$, è risultata efficace nel trattamento del disturbo d'ansia, dell'ansia da separazione e della fobia sociale, in un piccolo gruppo di soggetti giovani- adolescenti di 11-17 anni [145].

Uno studio pilota condotto in doppio cieco ha evidenziato un miglioramento della sintomatologia in un gruppo di pazienti adulti affetti da fobia sociale. Tuttavia, non è stato possibile dimostrare una differenza statisticamente significativa rispetto al placebo. Questo risultato può essere dovuto al basso numero di soggetti o alla posologia adottata (20mg/die per le prime 8 settimane con possibilità di aumentare fino a $60 \mathrm{mg} /$ die nelle ultime 6 settimane) [144].

\section{Bulimia nervosa}

La fluoxetina è efficace nel trattamento della bulimia nervosa alla dose di $60 \mathrm{mg} / \mathrm{die}$; dosi inferiori sono risultate inefficaci. Gli effetti terapeutici sono evidenti già dopo una settimana di trattamento, ma la terapia deve essere prolungata finché il successo non può essere considerato stabile. Durante la fase di mantenimento si deve verificare periodicamente la necessità di continuare il trattamento.

Un recente studio randomizzato in doppio cieco ha verificato l'efficacia a lungo termine della fluoxetina in un gruppo di pazienti affetti da bulimia nervosa di tipo purgativo, classificati come responders ad una fase iniziale di trattamento (riduzione di almeno il 50\% degli episodi di vomito durante almeno una delle ultime due settimane del periodo acuto di 8 settimane). I 150 responders (65\% dei 232 trattati in fase acuta) sono stati randomizzati un due gruppi, uno trattato con fluoxetina $60 \mathrm{mg} /$ die e l'altro con placebo. Durante i primi 3 mesi di mantenimento la frequenza di recidive è stata significativamente inferiore nel gruppo che ha continuato il trattamento con fluoxetina, nei mesi successivi la differenza di recidive tra fluoxetina e placebo si è stabilizzato attorno ad una differenza del 14-18\%. Al termine delle 52 settimane previste dal protocollo la percentuale di recidive era del $33 \%$ nel gruppo fluoxetina e del $51 \%$ nel gruppo placebo. Nel gruppo trattato con fluoxetina la presenza di depressione non influiva sulla frequenza delle recidive. $\mathrm{La}$ fluoxetina ha ridotto significativamente il numero di episodi di vomito e di binge-eating e migliorato i punteggi delle scale utilizzate per valutare il disturbo. La percentuale di abbandoni per reazioni avverse è stata analoga nei due gruppi, mentre il numero di abbandoni durante i primi 3 mesi per efficacia inferiore a quella attesa è stata superiore nel gruppo placebo [149].

L'effetto della fluoxetina sulla bulimia nervosa è indipendente dall' effetto antidepressivo, anche nei pazienti affetti comorbilità depressiva [150].

L'effetto della fluoxetina è selettivo sulla compulsività bulimica, mentre risulta inefficace sull'assunzione compulsiva di acqua [151].

La fluoxetina normalizza il tono vagale cardiaco che risulta elevato nelle donne affette da bulimia [152].

La terapia con fluoxetina aumenta la probabilità di successo della psicoterapia cognitiva, potenziando soprattutto il miglioramento del vomito, del binge-eating e della depressione $[153,154]$. In un piccolo gruppo di pazienti che non avevano risposto adeguatamente alla psicoterapia cognitiva si è registrato un miglioramento dopo un trattamento con fluoxetina per 8 settimane [155].

L'effetto della fluoxetina può essere potenziato da una strategia cognitiva basta sull'utilizzo di un manuale di autoaiuto [156].

\section{Dolore cronico}

Gli SSRI possono trovare indicazione anche nel trattamento di alcune forme di dolore cronico, come monoterapia o in associazione con gli analgesici maggiori. La fluoxetina è il farmaco più studiato con questa indicazione (dolore cronico, cefalea, dolore neuropatico, fibromialgia) e studi sperimentali recenti hanno confermato che l'effetto antalgico si esplica sia attraverso i circuiti serotoninergici e oppioidi sia probabilmente attraverso una azione periferica con inibizione dose-dipendente della sintesi di nitrossido e PGE2.

Recenti ricerche cliniche controllate versus placebo hanno dimostrato che la fluoxetina, a dosi variabili da 10 a $80 \mathrm{mg} / \mathrm{die}$, è in grado di controllare la sintomatologia dolorosa nelle donne che soffrono di fibromialgia [165].

In un piccolo gruppo di pazienti, sofferenti di dolori muscolo-scheletrici (dolori dorsali, 
cervicali), il trattamento con fluoxetina $20 \mathrm{mg} /$ die è stato efficace quanto il trattamento di controllo con amitriptilina 50-75 mg/die, inducendo un adeguato effetto analgesico in circa l'80\% dei casi [164].

Secondo alcuni autori, l'azione analgesica della fluoxetina nel dolore cronico, pur essendo equivalente come intensità e percentuale di successi a quella della fluvoxamina, si differenzierebbe da questa per essere strettamente correlata al miglioramento dei sintomi depressivi [163].

Controversi sono i risultati che riguardano l'efficacia analgesica della fluoxetina nelle cefalee. I dati disponibili indicano un moderato livello di efficacia nella cefalea cronica giornaliera e una mancanza di efficacia nel controllare i sintomi dell'emicrania $[167,168]$.

Tuttavia, ricerche condotte su piccoli gruppi di pazienti affetti da emicrania hanno dimostrato che la fluoxetina, somministrata a lungo termine, è efficace nella profilassi dell'emicrania [169]. Quest' attività sembra essere dovuta all'isomero S-fluoxetina.[168]

\section{Disfunzione sessuale: eiaculazione prematura}

La fluoxetina è efficace nel trattamento sintomatico della eiaculazione prematura. I risultati obiettivi (prolungamento da 1 a 6-10 minuti dell'intervallo pre-eiaculatorio intravaginale) si possono osservare dopo almeno due settimane di trattamento. Parallelamente aumenta la soddisfazione sessuale del soggetto e della partner, aumenta il desiderio sessuale e diminuisce l'ansia da prestazione. Tuttavia, il risultato sembra non essere duraturo e 2-3 settimane dopo la sospensione il disturbo recidiva in oltre il $90 \%$ dei soggetti [182-184].

Uno studio condotto in doppio cieco su gruppi di soggetti affetti da ejaculatio praecox isolata (PE) o accompagnata da disfunzione erettile (DE), ha evidenziato un significativo prolungamento della latenza pre-eiaculazione nell'insieme dei soggetti o nei soggetti con associata DE, ma un prolungamento statisticamente non significativo nei soggetti affetti solo da PE [185].

L'effetto della fluoxetina sulla eiaculazione prematura sarebbe potenziato dall' applicazione di una pomata alla lidocaina; tuttavia questo risultato dovrà essere confermato da ulteriori trial in doppio cieco [186].

L'effetto della fluoxetina sulla eiaculazione prematura sarebbe dovuto all'incremento della soglia di sensibilità del pene; l'ampiezza e la latenza della risposta sacrale evocata e dei potenziali evocati somatosensoriali corticali non sono modificati dalla fluoxetina [187].

\section{PROFILODITOLLERABILITÀ ESICUREZZA}

Il profilo di sicurezza della fluoxetina è descritto in alcune rassegne, in riferimento sia a trattamenti brevi che prolungati $[188,189]$.

Dall'esame dei dati disponibili emerge che la fluoxetina, per quanto riguarda la sicurezza, può vantare una minore incidenza di eventi avversi e una minore frequenza di drop-outs rispetto ai triciclici e agli altri antidepressivi SSRI; inoltre presenta un buon livello di sicurezza anche nei casi di sovradosaggio e il suo uso è sicuro in speciali sottopopolazioni di pazienti, come nelle donne in gravidanza.

Studi prospettici controllati, condotti su donne in gravidanza accertata, trattate con SSRI e soprattutto con fluoxetina, hanno evidenziato l'assenza di effetti teratogeni.

Un vantaggio peculiare della fluoxetina, correlabile alla sua lunga emivita e alla prolungata durata d' azione, è l'assenza di sintomi, o la presenza di sintomi molto sfumati, alla sospensione del trattamento.

Una ricerca condotta in doppio cieco versus placebo ha verificato la sicurezza della sospensione improvvisa della fluoxetina in un gruppo di pazienti in fase di mantenimento da 12 settimane. Il gruppo di pazienti passati al placebo non hanno manifestato eventi avversi qualitativamente e quantitativamente differenti dal gruppo che ha continuato la terapia con fluoxetina. La sospensione della fluoxetina è stata ben tollerata e non ha provocato l'insorgenza di eventi avversi di rilevanza clinica [190].

Questo risultato differenzia nettamente la fluoxetina rispetto agli altri SSRI e indica che l'uso della fluoxetina è preferibile per quei pazienti che hanno una bassa compliance posologica, dimenticano frequentemente di assumere la dose giornaliera o hanno difficoltà ad assumerla continuativamente per motivi pratici sociali (es. viaggi, ecc.).

\section{Reazioni avverse}

Il principale evento avverso correlato alla terapia con fluoxetina è la nausea. Altri eventi avversi sono rappresentati da ipotensione, cefalea, insonnia, ansia, secchezza delle fauci, anoressia e perdita di peso, disturbi visivi.

Gli SSRI, rispetto ai triciclici, inducono più frequentemente diarrea, cefalea, insonnia e nausea. I triciclici, invece, rispetto agli SSRI, inducono più frequentemente disturbi visivi, stipsi, vertigini, secchezza delle fauci, tremori e disturbi urinari.

Anche i disturbi della sfera sessuale sono frequenti, ma una stima realistica dell'incidenza delle reazioni avverse e un confronto quantitativo tra classi di antidepressivi appare 
impossibile per il basso numero di trial disponibili.

I nuovi antidepressivi, come i vecchi, possono indurre in rari casi alcune gravi reazioni avverse. Nove sono le reazioni avverse rare (incidenza $<1 \%$ ) ma gravi attribuite con certezza ai nuovi antidepressivi, e in particolare alla classe degli SSRI. Questo quadro di reazioni avverse rare è emerso progressivamente con la diffusione dei nuovi prodotti nell'uso clinico naturalistico.

La sindrome serotoninica è una condizione potenzialmente fatale caratterizzata da disturbi cognitivi, agitazione, miocloni, iperriflessia, diaforesi, brividi, tremori, incoordinazione motoria, febbre e diarrea. $\mathrm{Si}$ tratta di una sindrome sintomatologica simile alla ipertermia maligna o alla sindrome neurolettica maligna. Il rischio di sindrome serotoninergica aumenta se ai farmaci SSRI vengono associati IMAO o altri farmaci serotoninergici: la sindrome serotoninica è stata osservata nel $75 \%$ dei soggetti che avevano iniziato un trattamento con fluoxetina quando il paziente assumeva ancora un IMAO [191].

Rari casi di anemia aplastica, pancitopenia, neutropenia e disturbi emostatici sono stati segnalati in pazienti trattati con fluoxetina.
Una rilevante percentuale di reazioni avverse segnalate nel corso di un trattamento con fluoxetina sono in realtà da attribuire ad una qualche associazione farmacologica (vedi Tabella 4).

\section{Rischio di suicidio}

Due studi di coorte e sette rassegne di trial hanno evidenziato come nei soggetti trattati con SSRI vi sia una tendenziale riduzione del rischio di suicidio [192]. Questo risultato è stato confermato specificamente anche per la fluoxetina [193,194]. Un recente studio prospettico naturalistico di follow-up condotto su 643 pazienti e durato dal 1987 al 1996 ha evidenziato che il rischio di suicidio tendenzialmente diminuisce nei soggetti trattati con fluoxetina.

Il rischio di suicidio è correlato alla gravità della depressione e ad ogni tentativo di suicidio il rischio aumenta marginalmente, sia pure di poco [194].

In caso di tentativo di suicidio con overdose di antidepressivo, si ritiene che gli SSRI siano meno tossici e meno letali. Una casistica di avvelenamenti acuti con fluoxetina ha dimostrato una mortalità molto bassa [195].

\begin{tabular}{|c|c|c|c|}
\hline Reazione avversa & Farmaci & Incidenza \% & Livello di evidenza \\
\hline Convulsioni & $\begin{array}{l}\text { SSRI, } \\
\text { bupropione }\end{array}$ & $<0,05 \%-0,26 \%$ & $\begin{array}{l}\text { Tendenza all'aumento rispetto } \\
\text { ai controlli e correlazioni } \\
\text { dose-effetto in studi controllati }\end{array}$ \\
\hline Iposodiemia/SIADH & SSRI & $<1 \%$ & $\begin{array}{l}\text { Uno studio caso-controllo, } \\
\text { rassegna sistematica di casi } \\
\text { con positività al dechallenge e } \\
\text { rechallenge }\end{array}$ \\
\hline Sindrome serotoninica & $\begin{array}{l}\text { SSRI (overdose } \\
\text { o in associazione } \\
\text { con altri farmaci } \\
\text { serotoninergici) }\end{array}$ & & $\begin{array}{l}\text { Casi con positività al } \\
\text { dechallenge }\end{array}$ \\
\hline Effetti extrapiramidali & SSRI & $\begin{array}{l}0,4 \%-13 \% \\
\text { (in funzione di } \\
\text { fattori di rischio } \\
\text { sottostanti) }\end{array}$ & $\begin{array}{l}\text { Casi con positività al } \\
\text { dechallenge }\end{array}$ \\
\hline Mania in depressione unipolare & SSRI & $<1 \%$ & Meta-analisi significativa \\
\hline Bradicardia & SSRI & Ignota & $\begin{array}{l}\text { Segnalazione di casi, con un } \\
\text { paziente positivo al } \\
\text { rechallenge }\end{array}$ \\
\hline Sanguinamento & SSRI & $<0,73 \%$ & $\begin{array}{l}\text { Studio controllato, casi con } \\
\text { positività al rechallenge }\end{array}$ \\
\hline Granulocitopenia & SSRI & Ignota & $\begin{array}{l}\text { Caso con positività al } \\
\text { dechallenge e rechallenge }\end{array}$ \\
\hline Epatotossictà & SSRI & Ignota & $\begin{array}{l}\text { Casi con positività al } \\
\text { dechallenge }\end{array}$ \\
\hline
\end{tabular}




\section{Controindicazioni}

Oltre alla ipersensibilità nei confronti della molecola, la fluoxetina non dovrebbe essere somministrata prima di due settimane dalla sospensione della terapia con un farmaco inibitore delle monoaminossidasi.

Particolare attenzione deve essere posta per i pazienti con patologie epatiche poiché potrebbe essere richiesto un aggiustamento posologico dovuto alla preponderante quota metabolizzata a livello epatico.

La fluoxetina può alterare il controllo glicemico con la possibile comparsa all'inizio della terapia di ipoglicemia.

Particolare attenzione deve essere posta inoltre in caso di somministrazione in pazienti con anamnesi positiva per crisi convulsive e in caso di modificazioni del peso e dell' appetito e/o di comparsa di sintomi ansiosi, insonnia, mania o ipomania.

\section{PROFILOFARMACOECONOMICODEI PRODOTTI GENERICIDI FLUOXETINA}

I disturbi depressivi sono uno dei maggiori problemi di sanità pubblica per l'alta prevalenza, la ubiquitarietà e le fasce d'età coinvolte, per le gravi sofferenze inflitte ai pazienti e ai loro familiari e per gli oneri economici che la società deve sopportare [196].

L'Organizzazione Mondiale della Sanità (OMS) nel 1990 ha classificato la depressione come la quarta malattia più comune al mondo, destinata a passare al secondo posto entro il 2020 [197].

Il rischio di ammalarsi di depressione maggiore nell' arco della vita è stato stimato tra il $7 \%$ e il $12 \%$ per gli uomini e tra il $20 \%$ e il $25 \%$ per le donne [190-200].

I soggetti depressi presentano gradi di inabilità e di menomazione delle funzionalità individuali e sociali che sono spesso più gravi di quelle causate da altre malattie come l'ipertensione, la malattia coronarica, la bronchite cronica, il mal di schiena [201].

La depressione, interessando una vasta fascia di popolazione a partire fin dalla giovane età, induce sofferenze e costi per un periodo di tempo più lungo, ma con modalità più sfumate e subdole rispetto ad altre malattie di grande impatto sociale. Un carico economico notevole grava sui soggetti in età lavorativa e, più in generale, sul sistema produttivo a causa dell'inabilità e dei disturbi della funzionalità sociale che la depressione induce [202-205].

La depressione, inoltre, è correlata ad un elevato livello di mortalità, dovuto principalmente ai casi di suicidio e all'interazione con altre malattie. Oltre il $60 \%$ dei casi di suicidio riguarda soggetti affetti da disturbo depressivo maggiore.

All'inizio degli anni '90 il costo di malattia della depressione negli USA è stato stimato in oltre 52 miliardi di dollari/anno, corrispondente a circa 3.000 dollari/anno per ogni paziente [206].

Il costo totale della depressione è costituito per circa un quarto dai costi diretti sanitari e per i rimanenti tre quarti dai costi indiretti. Tra i costi diretti sanitari la spesa per terapie farmacologiche rappresenta solo circa il 12\%, mentre oltre la metà delle risorse sanitarie sono assorbite dai ricoveri per episodi acuti $(53 \%)$ e circa il $30 \%$ dall' assistenza psichiatrica extraospedaliera. I costi indiretti sono costituiti per il $19 \%$ dalla perdita di produttività dovuta a morti premature per suicidio, per il $60 \%$ da perdita di produttività per assenteismo dal lavoro e per il restante $21 \%$ da ridotta produttività sul lavoro.

La depressione, oggi, può essere curata in un' elevata percentuale di casi con farmaci efficaci e ben tollerati anche a lungo termine, come la fluoxetina [30,207,208].

L'uso di farmaci antidepressivi, oltre a ridurre il carico di sofferenze che grava sui pazienti depressi e sulle loro famiglie, può comportare una significativa riduzione di alcuni costi diretti sanitari, come il costo ospedaliero, e di alcuni costi indiretti. Sicché il maggior costo del trattamento farmacologico può essere in tutto o in parte controbilanciato dal maggior risparmio eventualmente inducibile sui costi diretti e indiretti.

Nella realtà clinica quotidiana ogni paziente depresso ha problemi e bisogni personali che devono essere affrontati dal medico curante in modo ottimale, sia per aumentare la probabilità di successo sia per ridurre al minimo i costi.

Per una esauriente analisi della relazione tra percorsi diagnostici-terapeutici del paziente depresso e formazione dei costi di un trattamento farmacologico si rimanda ad un recente lavoro [209].

Nella prospettiva generale di un decisore istituzionale, che deve ottimizzare l'utilizzo delle scarse risorse disponibili per ottenere il massimo beneficio per la collettività, valutare la convenienza di un farmaco significa attuare un' analisi costo/efficacia, mettendo a confronto costi e risultati delle alternative reali disponibili. Quando i trattamenti confrontati hanno efficacia equivalente, l' analisi costo/efficacia si limita alla valutazione e valorizzazione dei costi e all'applicazione dei criteri decisionali della tecnica nota come "minimizzazione dei costi", un sottotipo semplificato di analisi costo/efficacia. Nell'ambito dei trattamenti farmacologici della depressione, l'efficacia dei principali farmaci può essere considerata equivalente e pertanto più frequentemente viene usata la tecnica "minimizzazione dei costi". 
Sono considerati decisori primari la società, che rappresenta l'interesse dell'insieme dei pazienti, e il SSN, al quale viene attribuito il compito di provvedere all'assistenza sanitaria dei cittadini di una nazione. Altri decisori talvolta considerati sono l'ospedale e le assicurazioni (terzo pagante privato).

La tipologia del decisore e i suoi specifici interessi condizionano le voci di costo e i parametri di efficacia da considerare nell' ambito di un'analisi costo/efficacia. Gli interessi di alcuni decisori possono essere talvolta conflittuali, ma nella prospettiva più generale della società dovrebbero trovare un' armonica composizione.

Volendo esaminare la costo/efficacia di trattamenti antidepressivi nella prospettiva più generale della società, si dovrebbero valutare e valorizzare sia i costi diretti sia i costi indiretti indicati nella Tabella 7. Nel caso l'analisi costo/efficacia sia attuata nella prospettiva del SSN, si devono includere solo i costi diretti sanitari che sono a carico del SSN stesso, mentre si devono escludere tutti gli altri costi diretti e indiretti che gravano sul paziente e sui suoi familiari, oltre che sulle strutture produttive della società.

La costo/efficacia della fluoxetina nella depressione è stata oggetto di alcune valutazioni farmacoeconomiche pubblicate negli ultimi anni. Benché i risultati di tali valutazioni non siano stati sempre concordi a causa soprattutto delle differenti metodologie utilizzate, della differente prospettiva e del diverso arco temporale assunti, nonché dei diversi sistemi socio-assistenziali nei quali sono state collocate, la fluoxetina appare generalmente più vantaggiosa dei triciclici e altrettanto costo/efficace almeno quanto, se non di più, altri SSRI, in particolare quanto la sertralina e la paroxetina [210-215].

I risultati ottenuti in queste valutazioni farmacoeconomiche, condotte prevalentemen- te negli Stati Uniti, non sono direttamente trasferibili alla situazione italiana, caratterizzata dalla presenza di un SSN forte che, a differenza degli USA, garantisce un'ampia copertura generale dell' assistenza sanitaria alla popolazione.

La Tabella 7, avendo presente 1'attuale assetto del SSN italiano, differenzia le principali voci di costo di un trattamento antidepressivo, ripartendoli sul SSN, sul paziente e sulla società. Il tipo di organizzazione dell'assistenza sanitaria può comportare una diversa ripartizione degli oneri: ad esempio una riclassificazione dei farmaci rispetto alla rimborsabilità da parte del SSN o l'introduzione di ticket può modificare sensibilmente la ripartizione degli oneri tra paziente e SSN. La politica dei generici introdotta di recente in Italia prevede il rimborso da parte del SSN del solo prezzo di riferimento, mentre a carico del paziente pesa l'onere dell'eventuale differenza di prezzo del prodotto branded e, in alcune regioni, del ticket per confezione.

I prodotti generici, se confrontati con gli equivalenti prodotti di marchio, realizzano una strategia di minimizzazione dei costi. La convenienza ad usare un prodotto generico in sostituzione di un prodotto "originatore" è intrinseca al concetto stesso di prodotto generico e viene amplificata dall'attuale normativa sui generici adottata in Italia.

Quando un generico venga confrontato con il relativo originatore, l'analisi "minimizzazione dei costi" risulta notevolmente semplificata e può essere ridotta alla sola analisi comparativa dei costi d'acquisto.

I prodotti generici di fluoxetina sono stati $\mathrm{i}$ primi a comparire sul mercato italiano nell' ambito della classe degli SSRI. La recente riclassificazione delle specialità medicinali ai fini del Prontuario Terapeutico Nazionale, entrata in vigore all'inizio del 2003, ha fissato il incrementali attribuibili, come oneri, al Sistema Sanitario Nazionale, al paziente e alla società nell'ambito di un'analisi

"minimizzazione dei costi” [209]

Parz: contributo parziale da parte del paziente (ad esempio, alcuni farmaci non rimborsati o rimborsati parzialmente secondo prezzi di riferimento, come nel caso siano disponibili prodotti generici)

Ticket: compartecipazione alla spesa sotto forma di ticket

\begin{tabular}{lccc}
\hline COSTI INCREMENTALI & SSN & PAZ & SOC \\
\hline DIRETTI SANITARI & & & \\
Acquisto farmaco & SI & Parz & SI \\
Esami diagnostici indotti & SI & Ticket & SI \\
Visite mediche & SI & & SI \\
Visite Psichiatriche & SI & & SI \\
Ricoveri ospedalieri & SI & Parz & SI \\
Costi per eventi avversi & SI & & \\
DIRETTI NON SANITARI & & & \\
Assistenza complementare & & SI & SI \\
INDIRETTI & & & \\
Assenteismo da effetto farmacologico & & SI & SI \\
Ridotto rendimento da effetto farmacologico & & SI & SI \\
\hline
\end{tabular}




\begin{tabular}{lccc}
\hline Parametro & Branded & Generico $^{\circ}$ & Differenza* $^{\circ}$ \\
\hline Prezzo confezione al pubblico (2003) & & & \\
& & & \\
$12 \mathrm{cps} 20 \mathrm{mg}$ & $10,03-13,02$ & 4,5 & $5,8-8,52$ \\
$28 \mathrm{cps} 20 \mathrm{mg}$ & $23,1-28,72$ & 16,82 & $6,28-11,90$ \\
Costo acquisto farmaco & & & \\
Costo/die (20mg/die) & $0,83-1,09$ & 0,38 & $0,45-0,71$ \\
Costo/ciclo 8 settimane & $46,20-60,76$ & 21,00 & $25,20-39,76$ \\
\hline
\end{tabular}

\section{Tabella 8}

Prezzo delle confezioni orali dei prodotti branded e generici di fluoxetina disponibili in Italia e relativi costi di trattamento (acquisto) giornaleiro o per ciclo breve (PFN 2003)

* La differenza corrisponde al risparmio che può ottenere il paziente se accetta la sostituzione del prodotto branded prescritto da medico con il prodotto generico.

- Prezzo di riferimento rimborsato dal SSN

prezzo dei generici di fluoxetina come valore rimborsabile dal SSN.

Nella Tabella 8 sono riportate le confezioni orali solide di fluoxetina disponibili in Italia come prodotti branded e come equivalenti prodotti generici, il loro prezzo al pubblico, il costo di trattamento (costo d'acquisto) giornaliero alla dose abituale di $20 \mathrm{mg} /$ die e il costo di un ciclo di terapia di 8 settimane, nonché le differenze tra branded e generico.

Il prezzo del generico viene rimborsato dal SSN, mentre la differenza è a carico del paziente.Le confezioni dei prodotti generici di fluoxetina attualmente costano da 5,80 euro a 11,90 euro in meno rispetto ai relativi prodotti branded. Questa cifra è il valore che il paziente deve pagare al farmacista se non accetta la sostituzione del prodotto di marchio con il prodotto generico.

Il costo d'acquisto dell' antidepressivo dipende, oltre che dal costo della confezione e quindi di una compressa, anche dalla posologia giornaliera e dalla durata del ciclo di trattamento.

Se il paziente accetta di utilizzare la fluoxetina generico al posto del prodotto di marchio, può ottenere un risparmio giornaliero variabile da 0,45 euro a 1,94 euro; per un ciclo di 8 settimane, necessario mediamente per trattare un episodio di depressione, il risparmio del paziente può variare da un minimo di 25,20 euro ad un massimo di 108,48 euro.

\section{Il mercato degli antidepressivi e la quota "genericabile" della fluoxetina}

Il mercato dei farmaci antidepressivi è tuttora in forte espansione ed è dominato dalla classe degli SSRI, mentre i triciclici mantengono le loro posizioni, pur con una lieve flessione. In forte crescita è anche il mercato degli SNRI.

La disponibilità di prodotti generici di fluoxetina, acquistabili a basso prezzo, può contribuire a modificare il mercato degli SSRI e marginalmente anche quello degli altri antidepressivi, producendo un sensibile rispar- mio per il SSN e per i pazienti, senza riduzione dei benefici sanitari.

I primi generici di fluoxetina sono stati introdotti in Italia nel 2000, conquistando una modesta percentuale del mercato (circa $1,4 \%$ in volume e $1,1 \%$ in valore). Nei due anni successivi, tuttavia, i generici di fluoxetina hanno progressivamente guadagnato consistenti
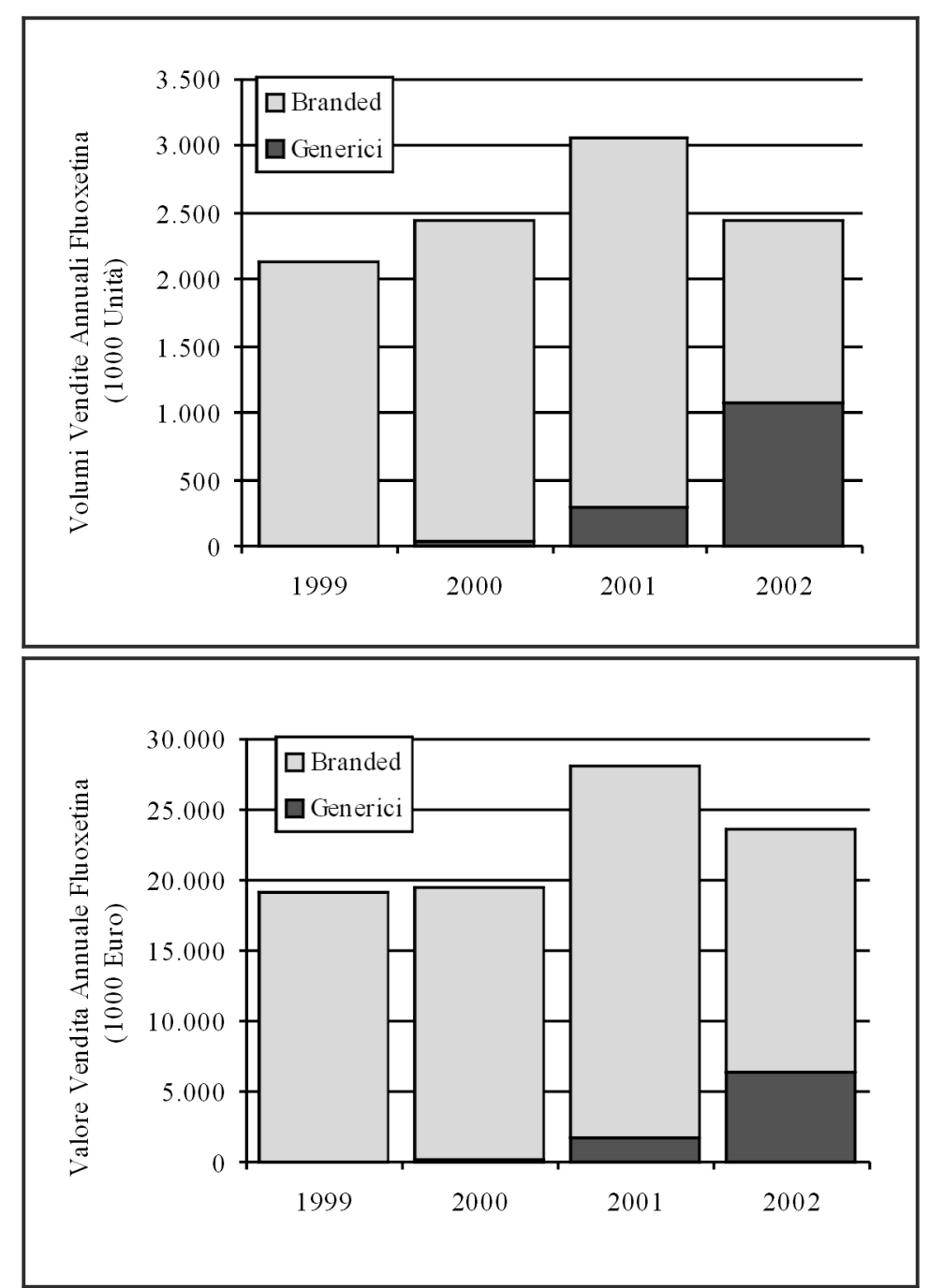

Figura 2

Vendite annuali di fluoxetina in Italia negli anni 1999-2002, espresse come volumi (sopra) e come valori al prezzo industriale (sotto). (Fonte: IMS) 
quote di mercato e nel 2002 rappresentavano circa il $44 \%$ del volume totale delle vendite di fluoxetina in Italia (il 27\% in valore) (Figura 2). Si noti che la riduzione del numero di confezioni di fluoxetina, e la parallela riduzione dei valori, registrata nel 2002 è dovuta non alla diminuzione del numero di pazienti ma all'introduzione di confezioni da 28 compresse o capsule, che hanno sostituito in gran parte le confezioni di 12 unità ancora disponibili.

La quota di mercato della fluoxetina teoricamente genericabile è ancora superiore al 50\% ed esistono, quindi, ancora margini per ulteriori significativi risparmi a vantaggio del SSN e dei pazienti, qualora si ampliasse l'uso dei prodotti generici di fluoxetina in sostituzione dei prodotti di marchio più costosi.

Inoltre, poiché la fluoxetina è equivalente come efficacia e tollerabilità agli altri SSRI, si può ipotizzare che in futuro un numero crescente di medici si convinca dell'opportunità di prescrivere i prodotti generici di fluoxetina a basso costo. Questa scelta potrà contribuire a ridurre il valore assoluto della spesa farmaceutica degli antidepressivi a carico del SSN.

Questo risultato può essere raggiunto solo con l'attiva collaborazione della classe medica e principalmente degli specialisti psichiatri e neurologi che determinano le linee di tendenza dell'utilizzo degli psicofarmaci.

\section{CONCLUSIONI}

La fluoxetina è stato il primo antidepressivo della classe degli inibitori selettivi della ricaptazione di serotonina (SSRI) ad essere sviluppato e commercializzato. Nonostante sia entrata nell'uso clinico da oltre quindici anni, la fluoxetina dimostra ancora una notevole vitalità e giovinezza e continua ad essere studiata in numerosi centri clinici in tutto il mondo sia come farmaco antidepressivo, sia come molecola efficace in numerose altre patologie psichiatriche e mediche che possono essere risolte o trovare giovamento dalla modulazione del sistema della serotonina. La presente rassegna ha evidenziato come, negli ultimi 6 anni, attorno alla fluoxetina vi sia stato un notevole fermento di nuove ricerche cliniche, che hanno portato ad una più approfondita conoscenza del suo valore terapeutico nelle depressioni, nei disturbi ossessivo-compulsivi e in numerosi disturbi d'ansia. Inoltre, alcune ricerche di notevole valore scientifico hanno prospettato la possibilità di impiegare la fluoxetina in nuove indicazioni, e in particolare nel disturbo disforico premestruale, nella bulimia nervosa, in alcune forme di dolore cronico e nel frequente disturbo sessuale della eiaculazione prematura.

La fluoxetina è una molecola ormai priva di protezione brevettuale per scadenza dei termini di legge e pertanto dal 2000 è disponibile sul mercato italiano anche come prodotto generico venduto ad un prezzo nettamente inferiore a quello dell' analogo prodotto di marchio.

Il prezzo dei prodotti generici orali di fluoxetina è attualmente il prezzo di riferimento rimborsato dal SSN. Pertanto, l'introduzione sul mercato italiano dei generici di fluoxetina ha consentito un significativo risparmio sulla spesa farmaceutica pubblica a beneficio del SSN e tale risparmio dovrebbe aumentare ancora nei prossimi anni.

I prodotti generici per definizione sono equivalenti per efficacia e tollerabilità agli analoghi prodotti di marchio che copiano. Pertanto il paziente che accetta la sostituzione del prodotto di marchio con il relativo generico è un paziente saggio perché, consapevole di non ricevere in cambio un prodotto di valore terapeutico inferiore, non è disposto a pagare la differenza di prezzo tra generico e branded solo per sentirsi protetto dal marchio.

\section{BIBLIOGRAFIA}

1. Stokes PE, Holtz A. Fluoxetine tenth anniversary update: the progress continues. Clin Ther 19: 1135-1250, 1997.

2. Eandi M. Ifarmaci "Generici”. Farmeconomia e Percorsi Terapeutici 2003; 4 (2): 65-76

3. Frazer A. Antidepressants. J Clin Psychiatry 58 (Suppl 6): 9-25, 1997.

4. Owens MJ, Morgan WN, Plott SJ, et al. Neurotransmitter receptor and transporter binding profile of antidepressants and their metabolites. J Pharmacol Exp Ther 283: 1305-1322, 1997.

5. Leonard BE, Richelson E. Synaptic effects of antidepressants. In "Schizophrenia and mood disorders: The new drug tehrapies in clinical practice. (Buckley, Waddington eds.), Butterworth-Heineman, Boston, 2000.

6. Lemberger L, Bergstrom RF, Wolen RL, et al. Fluoxetine: clinical pharmacology and physiologic disposition. J Clin Psychiatry 46: 14-19, 1985.

7. Saletu B, Grunberger J. Classification and determination of cerebral bioactivity offluoxetine: pharmacokinetic, pharmaco-eeg, and psychometric analyses. J Clin Psychiatry 46 (ePT2): 45-52, 1985. 
8. Aronoff GR, Bergstrom RF, Pottratz ST, et al. Fluoxetine kinetics and protein binding in normal and impaired renal function. Clin Pharmacol Ther 36: 138-144, 1984.

9. Chouinard G. A double-blind controlled clinical trial of fluoxetine and amitriptyline in the treatment of outpatients with major depressive disorder. J Clin Psychiatry 46: 32-37, 1985.

10. Liu ZQ, Cheng ZN, Huang SL, et al. Effect of the CYP2C19 oxidation polymorphism on fluoxetine metabolism in Chinese healthy subjects. J Clin Pharmacol 52: 96-99, 2001.

11. Harvey AT, Preskorn SH. Fluoxetine pharmacokinetics and effects on CYP2C19 in young and elderly volunteers. J Clin Psychopharmacol 21: 161-166, 2001.

12. Kristensen JH, Ilett KF, Hackett LP, et al. Distribution and excretion of fluoxetine and norfluoxetine in human milk. Br J Clin Pharmacol 48:521-527, 1999.

13. Taddio A, Ito S, Koren G. Excretion of fluoxetine and its metabolite, norfluoxetine, in human breast milk. J Clin Pharmacol 36 : 42-47, 1996.

14. Duffull SB, Begg EJ, Ilett KF. Fluoxetine distribution in human milk. J Clin Pharmacol 36: 1078-1079, 1996.

15. Schenker S, Bergstrom RF, Wolen RL, et al. Fluoxetine disposition and elimination in cirrhosis. Clin Pharmacol Ther 44: $353-$ $359,1988$.

16. Takemoto CK, Hodding JH, Kraus DM. Pediatric dosage handbook, 4th ed.,Lexi-Comp Inc., Hudson, OH, 1997.

17. Harvey AT, Preskorn SH. Cytochrome P450 enzymes: interpretation of their interactions with selective serotonin reuptake inhibitors. Part I. J Clin Psychopharmacol 16: 273-285, 1996.

18. Harvey AT, Preskorn SH. Cytochrome P450 enzymes: interpretation of their interactions with selective serotonin reuptake inhibitors. Part II. J Clin Psychopharmacol 16: 345-355, 1996.

19. Nemeroff CB, DeVAne CL, Pollock BG, et al. Newer antidepressants and the cytochrome P450 system. Am J Psychiatry 153: 311-320, 1996.

20. Liston HL, DeVAne CL, Boulton DW, et al. Differential time course of Cytochrome P450 2D6 enzyme inhibition by fluoxetine, sertraline, and paroxetine in healthy volunteers. J Clin Psychopharmacol 22: 169-173, 2002.

21. Stark P, Fuller RW \& Wong DT. The pharmacologic profile of fluoxetine. J Clin Psychiatry 46:7-13, 1985.

22. Stark P \& Hardison CD. A review of multicenter controlled studies of fluoxetine vs imipramine and placebo in outpatients with major depressive disorder. J Clin Psychiatry 46:53-58, 1985.

23. Fabre JF \& Crismon L. Efficacy of fluoxetine in outpatients with major depression. Curr Ther Res 37:115-123, 1985.

24. Rickels K, Amsterdam JD \& Avallone MF. Fluoxetine in major depression: a controlled study. Curr Ther Res 39:559-563, 1986.

25. Wernicke JF, Dunlop SR, Dornseif BE, et al. Fixed-dose fluoxetine therapy for depression. Psychopharmacol Bull 23:164-168, 1987.

26. Nierenberg AA, Farabaugh AH, Alpert JE, et al. Timing of onset of antidepressant response with fluoxetine treatment. Am J Psychiatry 157: 1423-1428, 2000.

27. Cohn JB \& Wilcox C. Comparison of fluoxetine, imipramine, and placebo in patients with major depressive disorder. J Clin Psychiatry 46:26-31, 1985.

28. Bremner JD. Fluoxetine in depressed patients: a comparison with imipramine. J Clin Psychiatry 45: 414-419, 1984.

29. Bech P, Cialdella P, Haugh MC, et al. Meta-analysis of randomised controlled trials of fluoxetine vs placebo and tricyclic antidepressants in the short-term treatment of major depression. Br J Psychiatry 176:421-428, 2000.

30. Williams JW, Mulrow CD, Chiquette E, et al. A systematic review of newer pharmacotherapies for depression in adults: evidence report summary. Ann Intern Med 132: 743-756, 2000.

31. Thompson C, Peveler RC, Stephenson D, et al. Compliance with antidepressant medication in the treatment of major depressive disorder in primary care : a randomized comparison of fluoxetine and a tricyclic antidepressant. Am J Psychiatry 157: 338-343, 2000.

32. Fava M, Amsterdam JD, Deltito JA, et al. A double-blind study of paroxetine, fluoxetine, and placebo in outpatients with major depression. Ann Clin Psychiatry 10: 145-150, 1998.

33. Sechter D, Troy S, Paternetti S, et al. A double-blind comparison of sertraline and fluoxetine in the treatment of major depressive episode in outpatients. Eur Psychiatry 14: 41-48, 1999.

34. Fava M, Hoog SL, Judge RA, et al. Acute efficacy of fluoxetine versus sertraline and paroxetine in major depressive disorder including effects of baseline insomnia. J Clin Psychopharmacol 22: 137-147, 2002.

35. Nurnberg HG, Thompson PM, Hensley PL. Antidepressant medication change in a clinical treatment setting: a comparison of the effectiveness of selective serotonine reuptake inhibitors. J Clin Psychiatry 60: 574-579, 1999.

36. Thase ME, Blomgren SL, Birkett MA et al. Fluoxetine treatment of patients with major depressive disorder who failed initial treatment with sertraline. J Clin Psychiatry; 58:16-21, 1997.

37. Reimherr FW, Amsterdam JD, Quitkin FM, et al. Optimal lenght of continuation therapy in depression : a prospettive assessment during long-term fluoxetine treatment. Am J Psychiatry 155: 1247-1253, 1998.

38. Gilaberte I, Montejo AL, De La Gandara J, et al. Fluoxetine in the prevention of depressive recurrencies: a double-blind study. J Clin Psychopharmacol 21: 417-424, 2001.

39. Burke WJ, Shelton H, McArthur-Miller, etal. Weekly dosing of fluoxetine for the continuation phase of treatment of major depression : results of a placebo-controlled, randomize clinical trial. J Clin Psychopharmacol 20: 423-427, 2000.

40. Burke WJ, McArthur-Miller DA. Exploring treatment alternatives: weekly dosing of fluoxetine for the continuation phase of major depressive disorder. J Clin Psychiatry; 62: 38-42, 2001.

41. Fava M, Rafanelli C, Grandi S, et al. Prevention of recurrent depression with cognitive therapy: preliminary findings. Arch Gen Psychiatry 55: 816-820, 1998.

42. Fava M, Rafanelli C, Grandi S, et al. Six-year outcome for cognitive behavioral treatment of residual symptoms in major depression. Am J Psychiatry 155: 1443-1445, 1998. 
43. Scott J, Teasdale JD, Paykel ES, et al. Effects of cognitive therapy on psychological symptoms and social functioning in residual depression. Br J Psychiatry 177: 440-446, 2000.

44. Perlis RH, Nierenberg AA, Alpert JE, et al. Effects of adding cognitive therapy to fluoxetine dose increase on risk of relapse and residual depressive symptoms in continuation treatment of major depressive disorder. J Clin Psychopharmacol 22: 474-480, 2002.

45. Reimherr FW, Strong RE, Marchant BK, et al. Factors affecting return of symptoms 1 year after treatment in a 62-week controlled study of fluoxetine in major depression. J Clin Psychiatry 22 (Suppl 6): 16-23, 2001.

46. McGrath PJ, Stewart JW, Petkova E, et al. Predictors of relapse during fluoxetine continuation or maintenance treatment of major depression. J Clin Psychiatry. 61 : 518-524, 2000.

47. Kroenke K, West SL, Swindle R, et al. Similar effectiveness of paroxetine, fluoxetine, and sertraline in primary care: a randomized trial. JAMA 286: 2947-2955, 2001.

48. Fava M, Rosenbaum JF, McGrath PJ, et al. A double-bkind, controlled study of lithium and tricyclic augmentation of fluoxetine in treatment resistant depression. Am J Psychiatry 151: 1372-1374, 1994.

49. Fava M, Alpert J, Nierenberg A, et al. Double-blind study of high-dose fluoxetine versus lithium or desipramine augmentation of fluoxetine in parzial responders and nonresponders to fluoxetine. J Clin Psychopharmacol 22: 379-387, 2002.

50. Smith WT, Londborg PD, Glaudin V et al. Short-term augmentation offluoxetine with clonazepam in the treatment of depression: a double-blind study. Am J Psychiatry; 155:1339-1345, 1998.

51. Perez V, Gilaberte I, Faries D et al. Randomised, double-blind, placebo-controlled trial of pindolol in combination with fluoxetine antidepressant treatment. Lancet 349:1594-1597, 1997.

52. Coppen A, Bailey J. Enhancement of the antidepressant action of fluoxetine by folic acid: a randomised, placebo-controlled trial. J Affect Disord 60: 121-130, 2000.

53. Small GW, Birkett M, Meyers BS, et al. Impact of physical illness on quality of life and antidepressant response in geriatric major depression. Fluoxetine Collaborative Group. J Am Ger Soc 44: 1120-1225, 1996.

54. Evans M, Hammond M, Wilson K, et al. Treatment of depression in the elderly: effect of physical illness on response. Int J Ger Psychiatry 12: 1189-1194, 1997.

55. Finkel SI, Richter EM, Clary CM, et al. Comparative efficacy of sertraline vs fluoxetine in patients age 70 or over with major depression. Am J Ger Psychiatry 7: 221.227, 1999.

56. Ackerman D, Greenand S, Bystritsky A, et al. Side effects and time course of response in a placebo-controlled trial offluoxetine for the treatment of geriatric depression. J clin Psychopharmacol 20: 658-665, 2000.

57. Cole MG, Elie LM, McCusker J, et al. Feasibility and effectiveness of treatmnets for depression in elderly medical inpatients: a systematic review. Int Psychogeriatrics 12: 453-461, 2000.

58. Newhouse PA, Krishnan KR, Doraiswamy PM, et al. A double-blind comparison of sertraline and fluoxetine in depressed elderly outpatients. J Clin Psychiatry. 61: 559-568, 2000.

59. Cassano GB, Puca F, Scapicchio PL, et al. Paroxetine and fluoxetine effects on mood and cognitive functions in depressed nondemented elderly patients. J clin Psychiatry 63: 396-402, 2002.

60. Yohannes AM, Connolly MJ, Baldwin RC. A feasibility study of antidepressant drug therapy in depressed elderly patients with chronic obstructive pulmonary disease. Int J Ger Psychiatry 16: 451-454, 2001.

61. Emslie GJ, Rush J, Weinberg WA et al. A double-blind, randomized, placebo-controlled trial of fluoxetine in children and adolescents with depression. Arch Gen Psychiatry; 54:1031-1037, 1997.

62. Emslie GJ, Heiligenstein JH, Wagner KD, et al. Fluoxetine for acute treatment of depression in children and adolescents: a placebo-controlled, randomized clinical trial. J Am Acad Child Adolesc Psychiatry 41: 1205-1215, 2002.

63. Kowatch RA, Carmody TJ, Emslie GJ, et al. Prediction of response to fluoxetine and placebo in children and adolescentys with major depression: a hypothesis generating study. J Affect Disord 54: 269-276, 1999.

64. Strober M, DeAntonio M, Schmidt-Lackner S, et al. The pharmacotherapy od depressive illness in adolescents: an open-label comparison of fluoxetine and imipramine-treated historical controls. J Clin Psychiatry 60: 164-169, 1999.

65. Dittmann RW, Czekalla J, Hundemer HP, et al. Efficacy and safety findings from naturalistic fluoxetine drug treatment in adolescent and young adult patients. J Child Adoles Psychopharmacol 10: 91-102, 2000.

66. Cornelius JR, Bukstein OG, Birmaher B, et al. Fluoxetine in adolescents with major depression and an alcohol use disorder : an open-label trial. Add Behav 26: 735-739, 2001

67. McGrath PJ, Stewart JW, Janal MN, et al. A placebo-controlled study of fluoxetine versus imipramine in the acute treatment of atypical depression. Am J Psychiatry 157: 344-350, 2000.

68. Appleby L, Warner R, Whitton A et al. A controlled study of fluoxetine and cognitive-behavioral counseling in the treatment of postnatal depression. BMJ; 314:932-936, 1997.

69. Ruhrmann S, Kasper S, Hawellek B et al. Effects of fluoxetine versus bright light in the treatment of seasonal affective disorder. Psychological Med; 28:923-933, 1998.

70. Ravindran AV, Bialik RJ , Lapierre YD. Therapeutic efficacy of specific serotonin reuptake inhibitors (SSRIs) in dysthymia. Can J Psychiatry; 39:21-26, 1994.

71. Albert R, Ebert D. Full efficacy of SSRI treatment in refractory dysthymia is achieved only after 16 weeks. J Clin Psychiatry; 57(4):176, 1996.

72. Vanelle JM, Attar-Levy D, Poirier MF et al. Controlled efficacy study of fluoxetine in dysthymia. Br J Psychiatry; 170:345$350,1997$.

73. Nobler MS, Devanand DP, Kim MK et al. Fluoxetine treatment of dysthymia in the elderly. J Clin Psychiatry; 57:254-256, 1996.

74. Waslick BD, Walsh BT, Greenhill LL, et al. Open trial of fluoxetine in children and adolescents with dysthymic disorder or double depression. J Affect Disord 56: 227-236, 1999.

75. Rickels K, Freeman EW, Sondheimer S, et al. Fluoxetine in the treatment of premenstrual syndrome. Curr Ther Res 48: 161-166, 1990. 
76. Steiner M, Steinberg S, Stewart D, et al. Fluoxetine in the treatment of premenstrual dysphoria .N Eng J Med 332: 1529-1534, 1995.

77. Steiner M, Romano SJ, Babcock S et al. The efficacy of fluoxetine in improving physical symptoms associated with premenstrual dysphoric disorder. Br J Obstet Gynaecol; 108:462-468, 2001.

78. Carr RR, Ensom MH. Fluoxetine in the treatment of premenstrual dysphoric disorder. Ann Pharmacother 36: 713-717, 2002.

79. Pearlstein T, Yonkers KA. Review of fluoxetine and its clinical applications in premenstrual dysphoric disorder. Exp Op Pharmacother 3: 979-991, 2002.

80. Hunter MS, Ussher JM, Browne SJ, et al. A randomized comparison of psychological (cognitive behavior therapy), medical (fluoxetine) and combined treatment for women with premenstrual dysphoric disorder. J Psychosom Obst Gyneco, 23: 193199, 2002.

81. Steiner M, Brown E, Trzepacz P, et al. Fluoxetine improves functional work capacity in women with premenstrual dysphoryc disorder. Arch Womens Ment Health 6: 71-77, 2003.

82. Connor KM, Sutherland SM, Tupler LA, et al. Fluoxetine in post-traumatic stress disorder. Randomized, double-blind study. Br J Psychiatry 175: 17-22, 1999.

83. Malik ML, Connor KM, Sutherland SM, et al. Quality of life and post-traumatic stress disorder: a pilot study assessing changes in SF-36 scores before and after treatment in a placebo-controlled trial of fluoxetine. J Traumatic Stress 12: 387-393, 1999.

84. Martenyi F, Brown EB, Zhang H, et al. Fluoxetine versus placebo in posttraumatic stress disorder. J Clin Psychiatry 63: 199206, 2002.

85. Martenyi F, Brown EB, Zhang $\mathrm{H}$, et al. Fluoxetine versus placebo in prevention of relapse in post-traumatic stress disorder. $\mathrm{Br}$ J Psychiatry 181: 315-320, 2002.

86. Cohen H, Kotker M,Matar M, et al. Normalization of heart rate variability in post-traumatic stress disorder patients following fluoxetine treatment: preliminary results. Israel Med As J 2: 296-301, 2000.

87. Barnett SD, Tharwani HM, Hertzberg MA, et al. Tolerability of fluoxetine in posttraumatic stress disorder. Prog NeuroPsychopahramcol Biol Psychiatry 26: 263-267, 2002.

88. Stamenkovic M, Blasbichler T, Riederer F, et al. Fluoxetine treatment in patients with recurrent brief depression. Int Clin Psychopharmacol 16: 221-226, 2001.

89. Volz HP, Laux P. Potential treatment for subtreshold and mild depression: a comparison of St. John's wort extracts and fluoxetine. Comp Psychiatry 41 (Suppl 1): 133-137, 2000.

90. Vercoulen JH, Swanink CM, Zitman FG et al. Randomised, double-blind, placebo-controlled study of fluoxetine in chronic fatigue syndrome. Lancet; 347:858-861, 1996.

91. Amsterdam JD, Garcia-Espana F, Fawcett J, et al. Efficacy and safety of fluoxetine in treating bipolar II major depressive episode. J Clin Psychopharmacol 18: 435-440, 1998.

92. Megna JL, Devitt PJ. Treatment of bipolar depression with twice-weekly fluoxetine. Management of antidepressant-induced mania. Annals Pharmacother 35: 45-47, 2001.

93. Sonawalla SB, Farabaugh A, Johnson MW, et al. Fluoxetine treatment of depressed patients with comorbid anxiety disorders. J Psychopharmacol 16:215-219, 2002.

94. Spalletta G, Pasini A, Caltagirone C. Fluoxetine alone in the treatment of first episodi anxious-depression: an open clinical trial. J Clin Psychopharmacol 22:263-266, 2002.

95. Versiani M, Ontiveros A, MAzzotti G, et al. Fluoxetine versus amitriptyline in the treatment of major depression with associated anxiety (anxious depression): a double-blind comparison. Int Clin Psychopharmacol 14: 321-327, 1999.

96. Fava M, Rosenbaum JF, Hoog SL, et al. Fluoxetine versus sertraline and paroxetine in major depression: tolerability and efficacy in anxious depression. J Aff Disorders 59: 119-126, 2000.

97. Cornelius JR, Salloum IM, Ehler JG, et al. Fluoxetine in depressed alcoholics. A double-blind, placebo-controlled trial. Arch Gen Psychiatry 54: 700-705, 1997.

98. Cornelius JR, Salloum IM, Thase Me, et al. Fluoxetine versus placebo in depressed alcoholic cocaine abusers. Psychopharmacol Bull 34: 117-121, 1998.

99. Cornelius JR, Salloum IM, Haskett RG, et al. Fluoxetine versus placebo in depressed alcoholics: a 1-year follow-up study. Add Behav 25: 307-310, 2000.

100. Cheer SM, Goa KL. Fluoxetine: a review of its therapeutic potential in the treatment of depression associated with physical illness. Drugs 61: 81-110, 2001.

101. Zisook S, Peterkin J, Goggin KJ et al. Treatment of major depression in HIV-seropositive men. J Clin Psychiatry; 59(5):217224,1998.

102. Ferrando SJ, Rabkin JG, de Moore GM et al. Antidepressant treatment of depression in HIV-seropositive women. J Clin Psychiatry; 60:741-746, 1999.

103. Fish MJ, Loeher PJ, Kristeller J, et al. Fluoxetine versus placebo in advanced cancer outpatients: a double-blind trial of the Hoosier Oncology Group. J Clin Oncol 21: 1937-1943, 2003.

104. Lustman PJ, Freedland KE, Griffith LS et al. Fluoxetine for depression in diabetes: a randomized double-blind placebocontrolled trial. Diabetes Care; 23(5):618-623, 2000.

105. Goodnick PJ. Use of antidepressants in treatment of comorbid diabetes mellitus and depression as well as in diabetic neuropathy. Annals Clin Psychiatry 13: 31-41, 2001.

106. Roose SP, Glassman AH, Attia E, et al. Cardiovascular effects of fluoxetine in depressed patients with heart disease. Am J Psychiatry 155: 660-665, 1998.

107. Amsterdam JD, Garcia-Espana F, Fawcett J, et al. Blood pressure changes during short-term fluoxetine treatment. J Clin Psychopharmacol 19: 9-14, 1999.

108. Strik JJM, Honig A, Lousberg R, et al. Efficacy and safety of fluoxetine in the treatment of patients with major depression after myocardial infarction: findings from a double-blind, placebo-controlled trial. Paychosom Med 62: 783-789, 2000. 
109. Dam M, Tonin P, De Boni A, et al. Effects offluoxetine an maproptiline on functional recovery in post-stroke hemiplegic patients undergoing rehabilitation therapy. Stroke 27: 1211-1214, 1996.

110. Wiart L, Petit H, Joseph PA, et al. Fluoxetine in early poststroke depression: a double-blind placebo-controlled study. Stroke 31: 1829-1832, 2000.

111. Robinson RG, Schultz SK, Castillo C, et al. Nortriptyline versus fluoxetine in the treatment of depression and in short-term recovery after stroke: a placebo-controlled, double-blind study. Am J psychiatry 157: 351-359, 2000.

112. Paolucci S, Antonucci G, Grasso MG, et al. Post-stroke depression, antidepressant treatment andrehabilitation results. A casecontrol study. Cerebrovasc Dis 12: 264-271, 2001.

113. Narushima K, Kosier JT, Robinson RG. Preventing poststroke depression: a 12-wek double-blind randomized treatment trial and 21-month follow-up. J Nerv Ment Dis $190: 296-303,2002$.

114. Fruehwald S, Gatterbauer E, Rehak P, et al. Early fluoxetine treatment of post-stroke depression. J Neurol 250: 347-351, 2003.

115. Petracca GM, Chemerinski E, Starkstein SE. A double-blind, placebo-controlled studyof fluoxetine in depresed patients with Alzheimer's disease. Int Psychogeriatrics 13: 233-240, 2001.

116. Turner SM, Jacob RG, Beidel DC et al. Fluoxetine treatment of obsessive-compulsive disorder. J Clin Psychopharmacol; 5:207212,1985 .

117. Jenike MA, Buttolph L, Baer Let al. Open trial of fluoxetine in obsessive-compulsive disorder. Am J Psychiatry; 146:909-911, 1989.

118. Levine R, Hofman JS, Knepple ED et al. Long-term fluoxetine treatment of a large number of obsessive-compulsive patients. J Clin Psychopharmacol; 9:281-283, 1989.

119. Browne M, Horn E, Jones TT. The benefits of clomipramine-fluoxetine combination in obsessive-compulsive disorder. Can J Psychiatry; 38:242-243, 1993.

120. Flament MF, Bisserbe JC. Pharmacologic treatment of obsessive-compulsive disorder: comparative studies. J Clin Psychiatry; 58(Suppl 12):18-22,1997.

121. Leonard HL. New developments in the treatment of obsessive-compulsive disorder. J Clin Psychiatry; 58(Suppl 14):39-45, 1997.

122. TodorovC, Freeston MH, Borgeat F. On the pharmacotherapy of obsessive-compulsive disorder: is a consensus possible? Can J Psychiatry 45: 257-262, 2000.

123. Fontaine R, Chouinard G. Fluoxetine in the long-term maintenance treatment of obsessive compulsive disorder. Psychiatr Ann; 19:88-91, 1989.

124. Ackerman DL, Greenland S, Bystritsky A. Clinical characteristics of response to fluoxetine treatment of obsessive-compulsive disorder. J Clin Psychopharmacol 18: 185-192, 1998.

125. Millet B, Touitou Y, Poirier MF, et al. Obsessive-compulsive disorder: evaluation of clincial and biological circadian parametres during fluoxetine treatment. Psychopharmacol 146: 268-274, 1999.

126. Rasmussen SA, Eisen JL. Treatment strategies for chronic and refractory obsessive-compulsive disorder. J Clin Psychiatry; 58(Suppl 13):9-13, 1997.

127. Romano S, Goodman W, Tamura R, et al. Long-term treatment of obsessive-compulsive disorder after an acute response: a comparison of fluoxetine versus placebo. J Clin Psychopharmacol 21: 46-52, 2001.

128. Maina G, Albert U, Bogetto F. Relapses after discontinuation of drug associated with increased resistance to treatment in obsessive-compulsive disorder. Int Clin psychopharmacol 16: 33-38, 2001.

129. Kurlan R, Como PG, Deeley C et al. Study of fluoxetine for obsessive-compulsive symptoms in children with Tourette's syndrome. Clin Neuropharmacol; 16:167-172, 1993.

130. Geller DA, Biederman J, Reed ED et al. Similarities in response to fluoxetine in the treatment of children and adolescents with obsessive-compulsive disorder. J Am Acad Child Adolesc Psychiatry; 34:36-44,1995.

131. Geller DA, Hoog SL, Heiligenstein JH, et al. Fluoxetine treatment for obsessive-compulsive disorder in children and adolescents: a placebo-controlled clinical trial. J Am Acad Child Adolesc Psychiatry 40: 773-779, 2001.

132. Liebowitz MR, Turner SM, Piacentini J, et al. Fluoxetine in children and adolescents with OCD: a placebo-controlled trial. J Am Acad Child Adolesc Psychiatry 41: 1431-1438, 2002.

133. Bloch MR, Elliott M, Thompson $\mathrm{H}$ et al. Fluoxetine in pathologic skin-picking. Open-label and double-blind results. Psychosomatics; 42:314-319, 2001.

134. Streichenwein SM, Thornby JI. A long-term, double-blind, placebo-controlled crossover trial of the efficacy of fluoxetine for trichotillomania. Am J Psychiatry; 152:1192-1196, 1995.

135. Sovner R, Fox CJ, Lowry MJ et al. Fluoxetine treatment of depression and associated self-injury in two adults with mental retardation. J Intellect Disabil Res; 37:301-311, 1993.

136. Ricketts RW, Goza AB, Ellis CR et al. Fluoxetine treatment of severe self-injury in young adults with mental retardation. J Am Acad Child Adolesc Psychiatry; 32(4):865-869, 1993.

137. Sheika SH, Wagner KD, Wagner RF. Fluoxetine treatment of trichotillomania and depression in a prepubertal child. Cutis; 51:5052, 1993.

138. McElroy SL, Kick PE, Pope HG, et al. Pharmacological treatment of kleptomania and bulimia nervosa. J Clin Psychopharmacol 9: 358-360, 1989.

139. Gorman JM, Liebowitz MR, Fyer AJ et al. An open trial of fluoxetine in the treatment of panic attacks. J Clin Psychopharmacol; 7:329-332, 1987.

140. Michelson D, Lydiard RB, Polack MH, et al. Outcome assessment and clinical improvement in panic disorder: evidence from a randomized controlled trial of fluoxetine and placebo. Am J Psychiatry 155: 1570-1577, 1998.

141. Michelson D, Polack MH, Lydiard RB, et al. Continuing treatment of panic disorder after acute response : randomized, placebo-controlled trial with fluoxetine. The Fluoxetine Panic Disorder Group. Br J Psychiatry 174: 213-218, 1999. 
142. Emmanuel NP, Ware MR, Brawman-Mintzer O et al. Once-weekly dosing of fluoxetine in the maintenance of remission in panic disorder. J Clin Psychiatry; 60:299-301, 1999.

143. Pallanti S, Quercioli L, Rossi A et al. The emergence of social phobia during clozapine treatment and its response to fluoxetine augmentation. J Clin Psychiatry; 60:819-823, 1999.

144. Kobak KA, Greist JH, Jefferson JW, et al. Fluoxetine in social phobia: a double-blnd, placebo-controlled pilot study. J Clin Psychopharmacol 22: 257-262, 2002.

145. Birmaher B, Waterman GS, Ryan N et al. Fluoxetine for childhood anxiety disorders. J Am Acad Child Adolesc Psychiatry; 33:993-999, 1994.

146. Barrickman L., Noyes R., Kuperman S. et al. Treatment of ADHD with fluoxetine: a preliminary trial. Journal of the American Academy of Child, Adolescent Psychiatry; 30(5):762-7, 1991.

147. Freeman CPI, Hampson M. Fluoxetine as a treatment for bulimia nervosa. Int J Obesity; 11(Suppl 3):171-177, 1987.

148. Marcus MD, Wing RR, Ewing L et al. A double-blind, placebo-controlled trial of fluoxetine plus behavior modification in the treatment of obese binge-eaters and non-binge-eaters. Am J Psychiatry; 147:876-881, 1990.

149. Romano SJ, Halmi KA, Sarkar NP et al. A placebo-controlled study of fluoxetine in continued treatment of bulimia nervosa after successful acute fluoxetine treatment. Am J Psychiatry; 159(1):96-102, 2002.

150. Goldstein DJ, Wilson MG, Ascroft RC, et al. Effectiveness of fluoxetine therapy in bulimia nervosa regardless of comorbid depression. Int J Eat Disord 25: 19-27, 1999.

151. Konreich C, Dan B, Verbank P, et al. Divergent responses o fluoxetine from two compulsive, food-related conditions: bulimia nervosa and compulsive water drinking. Biol Psychiatry 43: 310-311, 1998.

152. Rissanen A, Nakkarunen H, Virkkunen M, et al. Fluoxetine normalized increase cardiac vagal toe in bulimia nervosa. J Clin Psychopharmacol 18: 26-32, 1998.

153. Goldbloom DS, Olmsted M, Davis R, et al. A randomized controlled trial of fluoxetine and cognitive behavioral therapy for bulimia nervosa: short-term outcome. Beh Res Ther 35: 803-911, 1997.

154. Walsh BT, Wilson GT, Loeb KL et al. Medication and psychotherapy in the treatment of bulimia nervosa. Am J Psychiatry; 154:523-531, 1997.

155. Walsh BT, Agras WS, Devlin MJ, et al. Fluoxetine for bulimia nervosa following poor response to psychotherapy. Am J Psychiatry 157: 1332-1334, 2000.

156. Mitchell JE, Flechter L, Hanson K, et al. The relative efficacy of fluoxetine and manual-based self-help in the treatment of outpatients with bulimia nervosa. J Clin Psychopharmacol 21: 298-304, 2001.

157. Attia E, Haiman C, Walsh BT et al: Does fluoxetine augment the inpatient treatment of anorexia nervosa? Am J Psychiatry; 155:548-551,1998.

158. Phillips KA, Albertini RS, Rasmussen SA. A randomized placebo-controlled trial of fluoxetine in body dysmorphic disorder. Arch Gen Psychiatry $59: 381-388,2002$.

159. DeLong GR, Teague LA, McSwain Kamran M. Effects of fluoxetine treatment in young children with idiopathic autism. Develop Med Child Neurol. 40: 551-62, 1998.

160. Spina E, De Domenico P, Ruello C et al. Adjunctive fluoxetine in the treatment of negative symptoms in chronic schizophrenic patients. Int Clin Psychopharmacol; 9:281-285, 1994.

161. Poyurovsky M, Pashinian A, Gil-Ad I et al. Olanzapine-induced weight gain in patients with first-episode schizophrenia: a double-blind, placebo-controlled study of fluoxetine addition. Am J Psychiatry; 159(6):1058-1060, 2002.

162. Ratliff NB , Kerski D. Depersonalization treated with fluoxetine (Letter). Am J Psychiatry; 152:1689-1690, 1995.

163. Ciaramella A, Grosso S, Poli P. Fluoxetine versus fluovoxamine for treatment of chronic pain. Minerva Anest 66: 55-61, 2000.

164. Schreiber S, Vinokur S, Shavelzon V, et al. A randomized trial of fluoxetine versus amitriptyline in muscolo-scheletal pain. Israel J Psychiatry Rel Sci 38: 88-94, 2001.

165. Arnold LM, Hess EV, Hudson JL, et al. A randomized, placebo-controlled, double-blind, flexible-dose study offluoxetine in the treatment of women with fibromyalgia. Am J Med 112: 191-197, 2002.

166. Shemen L. Fluoxetine for treatment of tinnitus (Letter). Otolaryngol Head Neck Surg; 421, 1998.

167. Saper JR, Silberstein SD, Lake AE III et al. Double-blind trial of fluoxetine: chronic daily headache and migraine. Headache; 34:497-502, 1994.

168. Steiner TJ, Ahmed F, Findley LJ et al. S-fluoxetine in the prophylaxis of migraine: a phase II double-blind randomized placebocontrolled study. Cephalalgia; 18:283-286, 1998.

169. D'Amato CC, Pizza V, Marmolo T, et al. Fluoxetine for migraine prophylaxis: a double-blind trial. Headache 39: 716-719, 1999.

170. Loprinzi CL, Sloan JA, Perez EA et al. Phase III evaluation of fluoxetine for treatment of hot flashes. J Clin Oncol; 20(6):15781583, 2002.

171. Brown KW, Sloan RL, Pentland B. Fluoxetine as a treatment for post-stroke emotionalism. Acta Psychiatr Scand; 98:455-458, 1998.

172. Low BL, Chong SA. Treating poststroke pathologic crying with fluoxetine (Letter). J Clin Psychopharmacol; 18(3):252, 1998.

173. Pariente J, Loubinoux I, Carel C et al. Fluoxetine modulates motor performance and cerebral activation of patients recovering from stroke. Ann Neurol; 50:718-729, 2001.

174. Coleiro B, Marshall SE, Denton CP et al. Treatment of Raynaud's phenomenon with the selective serotonin reuptake inhibitor fluoxetine. Rheumatology; 40:1038-1043, 2001.

175. Sauer WH, Berlin JA, Kimmel SE. Selective serotonin reuptake inhibitors and myocardial infarction. Circulation; 104:18941898, 2001.

176. Levine LR, Rosenblatt S, Bosomworth J. Use of a serotonin re-uptake inhibitor, fluoxetine, in the treatment of obesity. Int $\mathrm{J}$ Obesity; 11(Suppl 3):185-190, 1987. 
177. Ferguson JM, Feighner JP. Fluoxetine-induced weight loss in overweight non-depressed humans. Int J Obesity 11 (Suppl 3): 163-170, 1987.

178. Goldstein DJ, Rampey AH, Enas GG et al. Fluoxetine: a randomized clinical trial in the treatment of obesity. Int J Obesity; 18:129-135, 1994.

179. Favale E, Rubino V, Mainardi P et al. Anticonvulsant effect of fluoxetine in humans. Neurology; 45:1926-1927, 1995.

180. Como PG, Rubin AJ, O'Brien CF et al. A controlled trial of fluoxetine in nondepressed patients with Huntington's disease. Mov Disord; 12:397-401, 1997.

181. Theesen KA, Marsh WR. Relief of diabetic neuropathy with fluoxetine. DICP; 23:572-574,1989.

182. Kara H, Aydin S, Yucel M et al. The efficacy of fluoxetine in the treatment of premature ejaculation: a double-blind placebo controlled study. J Urol; 156:1631-1632, 1996.

183. Lee HS, Song DH, Kim CH et al. An open clinical trial of fluoxetine in the treatment of premature ejaculation. J Clin Psychopharmacol; 16:379-382, 1996.

184. Kindler S, dolberg OT, Cohen H, et al. The treatment of comorbid premature ejaculation and panic disorder with fluoxetine. Clin Neurosychopharmacol 20: 466-471, 1997.

185. Haensel SM, Klem TMA, Hop WCJ, et al. Fluoxetine and premature ejaculation: a double-blind, crossover, placebo-controlled study. J Clin Psychopharmacol 18: 72-77, 1998.

186. Atan A, Basar MM, Aydoganli L. Comparison of the efficacy of fluoxetine alone vs fluoxetine plus local lidocaine oitmnet in the treatment of premature ejaculation. Arch Espan Urol 53 856-858, 2000.

187. Yilmaz U, Tatlisen A, turan $\mathrm{H}$, et al. The effects of fluoxetine on several neurophysiological variables in patients with premature ejaculation. J Urol 161: 107-111, 1999.

188. Wernicke JF. The side effect profile and safety of fluoxetine. J Clin Psychiatry 46(3 Pt 2):59-67, 1985.

189. Calil HM. Fluoxetine: a suitable long-term treatment. J Clin Psychiatry 22 (Suppl 22): 24-29, 2001.

190. Zajecka J, Fawcett J, Amsterdam J, et al. Safety of abrupt discontinuation of fluoxetine: a randomized, placebo-controlled study. J Clin Psychopharmacol 18: 193-197, 1998.

191. Feighner JP, Boyer WF, Tyler DL, Neborsky RJ. Adverse consequences of fluoxetine-MAOI combination therapy. J Clin Psychiatry 51:222-5, 1990.

192. Jick S, Dean AD, Jick H. Antidepressants and suicide. BMJ 310: 215-218, 1995.

193. Warshaw MG, Keller MB. The relationship between fluoxetine use and suicidal behaviour in 654 subjects with anxiety disorders. J Clin Psychiatry 57: 158-166, 1996.

194. Leon AC, Keller MB, Warshaw MSS, et al. Prospective study of fluoxetine treatment and suicidal behaviour in affectively ill subjects. Am J Psychiatry 156: 195-201, 1999.

195. Borys DJ, Setzer SC, Ling LJ, et al. Acute fluoxetine overoses: a report of 234 cases. Am J Emerg Med 10: 115-120, 1992.

196. Sartorius N. The economic and social burden of depression. J Clin Psychiatry 62 (suppl 15): 8-11, 2001.

197. Murray CJ, Lopez AD. The global burden of disease. Cambridge, Mass, Harvard University Press, 1996.

198. Johnson J, Weissman MM, Klerman GL. Service utilization and social morbidity associated with depressive symptoms in the community. JAMA 267: 1478-1483, 1992.

199. Klinkman MS. Competing demands in psychosocial care: a model for the identification and treatment of depressive disorders in primary care. Gen Hosp Psychiatry 19: 98-111, 1997.

200. Doris A, Ebmeier K, Shajahan P. Depressive illness. The Lancet 354: 1369-1375, 1999.

201. Wells KB, Stewart A, Hays RD, et al. The functioning and well-being of depressed patients: results from the Medical Outcomes Study. JAMA 262: 914-919, 1989.

202. Conti DJ, Burton WN. The economic impact of depression in a workplace. J Occupat Med 36: 983-988, 1994.

203. Kessler RC, Frank RG. The impact of psychiatric disorders on work loss days. Psychol Med 27: 861-873, 1997.

204. Druss BG, Rosenheck RA, Sledge WH. Health and disability costs of depressive illness in a major U.S. corporation. Am J Psychiatry 157: 1274-1278, 2000.

205. Goldberg RJ, Steury S. Depression in the workplace: costs and barriers to treatment. Psychiatric services 52: 1639-1643, 2001.

206. Greenberg PE, Stiglin LE, Finkelstein SN, Berndt ER. The economic burden of depression in 1990. J Clin Psychiatry. 54:405-18, 1993.

207. Mulrow CD, Williams JW, Jr., Chiquette E, et al. Efficacy of newer medications for treating depression in primary care patients. Am J Med 108: 54-64, 2000.

208. Snow V, Lascher S, Mottur-Pilson C; for the American College of Physicians-American Society of Internal Medicine. Pharmacologic Treatment of Acute Major Depression and Dysthymia: Clinical Guideline, Part 1. Ann Intern Med 132: 738-742, 2000.

209. Eandi M, Mencacci C, Torta R, et al. Farmacoeconomia e percorsi diagnostico-terapeutici del paziente depresso. Farmeconomia e Percorsi Terapeutici 3 (Suppl 1): 1-80, 2002.

210. Wilde MI, Benfield P. Fluoxetine. A pharmacoeconomic review of its use in depression. Pharmacoeconomics 13: 543-561, 1998.

211. Boyer P, Danion JM, Bisserbe JC, et al. Clinical and economic comparison of sertraline and fluoxetine in the treatment of depression. A 6-month double-blind study in a primary-care setting in France. Pharmacoeconomics 13: 157-169, 1998.

212. Russell JM, Berndt ER, Miceli R, et al. Course and cost of treatment fro depression with fluoxetine, paroxetine, and sertraline. Am J Mang Care 5: 597-606, 1999.

213. Fremantle N, Mason J. The importance of achieveing additional drug benefits at a reasonable cost. A review of the fluoxetine years. Pharmacoeconomics 17: 319-324, 2000.

214. Crown WH, Treglia M, Meneades L, et al. Long-term costs of treatment for depression: impact of drug selection and guideline adherence. Value in Health 4: 295-307, 2001.

215. Polsky D, Onesirosan P, Bauer MS, et al. Duration of therapy and health care costs of fluoxetine, paroxetine, and sertrlaine in6 health plans. J Clin Psychiatry 63: 156-164, 2002. 